\title{
Spatio-temporal Complexity of Slip on a Fault
}

\author{
JAMES R. RicE \\ Department of Earth and Planetary Sciences and Division of Applied Sciences, Harvard University, Cambridge, Massachusetts
}

\begin{abstract}
Three-dimensional analyses are reported of slip on a long vertical strike-slip fault between steadily driven elastic crustal blocks. A rate- and state-dependent friction law governs motion on the fault; the law includes a characteristic slip distance $L$ for evolution of surface state and slip weakening. Because temperature and normal stress vary with depth, frictional constitutive properties (velocity weakening/ strengthening) do also. Those properties are taken either as uniform along-strike at every depth or as perturbed modestly from uniformity. The governing equations of quasi-static elasticity and frictional slip are solved on a computational grid of cells as a discrete numerical system, and a viscous radiation damping term is included to approximately represent inertial control of slip rates during earthquakelike instabilities. The numerical results show richly complex slip, with a spectrum of event sizes, when solved for a grid with oversized cells, that is, with cell size $h$ that is too large to validly represent the underlying continuous system of equations. However, in every case for which it has been feasible to do the computations (moderately large $L$ only), that spatio-temporally complex slip disappears in favor of simple limit cycles of periodically repeated large earthquakes with reduction of cell size $h$. Further study will be necessary to determine whether a similar transition occurs when the elastodynamics of rupture propagation is treated more exactly, rather than in the radiation damping approximation. The transition from complex to ordered fault response occurs as $h$ is reduced below a theoretically derived nucleation size $h^{*}$ which scales with $L$ but is $2 \times 10^{4}$ to $10^{5}$ larger in cases considered. Cells larger than $h^{*}$ can fail independently of one another, whereas those much smaller than $h^{*}$ carinot slip unstably alone and can do so only as part of a cooperating group of cells. The results contradict an emergent view that spatio-temporal complexity is a generic feature of mechanical fault models. Such generic complexity does apparently result from models which are inherently discrete in the sense of having no well-defined continuum limit as $h$ diminishes. Those models form a different class of dynamical systems from models like the present one that do have a continuum limit. Strongly oversized cells cause the model developed here to mimic an inherently discrete system. It is suggested that oversized cells, capable of failing independently of one another, may crudely represent geometrically disordered fault zones, with quasi-independent fault segments that join one another at kinks or jogs. Such geometric disorder, at scales larger than $h^{*}$, may force a system with a well-defined continuum limit to mimic an inherently discrete system and show spatio-temporally complex slip at those larger scales.
\end{abstract}

\section{INTRODUCTION}

Slip histories $\delta(x, z, t)$ over repeated earthquake cycles are reported for a model with laboratory-based rate- and state-dependent friction along a long vertical strike-slip fault in an elastic half-space (Figure 1). The fault is driven by imposing slip at a uniform rate, equal to the long-term plate velocity $V_{\text {plate }}$, along the downward continuation of the fault plane into the half-space, i.e., on $-\infty<z<-Z_{\text {depth }}$. Solutions for $\delta(x, z, t)$ are determined over $-Z_{\text {depth }}<z<$ 0 and are restricted to be periodic in $x$ over an imposed period $X_{\text {length }}$. Because temperature and normal stress vary with depth, frictional constitutive properties do also. The calculations are based on constitutive data for velocity weakening or strengthening as a function of temperature in granite under hydrothermal conditions [Blanpied et al., 1991] and consider the possibility of either hydrostatic or elevated pore pressure conditions. Constitutive properties are taken either as uniform along-strike at every depth or perturbed modestly from uniformity to promote any latent instabilities.

The stress history $\tau(x, z, t)$ on the fault should be related to the slip history $\delta(x, z, t)$ there in a manner determined by solving the equations of continuum elastodynamics in the quarter spaces which adjoin along the fault. Given the interest here in the character of repeated earthquake re-

Copyright 1993 by the American Geophysical Union.

Paper number 93JB00191.

0148-0227/93/93JB-00191\$05.00 sponse based on a realistic friction law, solution of the full elastodynamic equations is regarded as too challenging computationally at the present time. Instead, the following radiation damping approximation is adopted: $\tau(x, z, t)$ is related to $\delta(x, z, t)$ by the equations of quasi-static equilibrium elasticity, but a viscous term $-\eta \partial \delta(x, z, t) / \partial t$ is added to the expression for $\tau(x, z, t)$. That term, with viscosity $\eta$ $=\mu / 2 c$ ( $\mu$ is elastic shear modulus, $c$ is shear wave speed), becomes significant only at very high slip rates $\partial \delta / \partial i$ and represents energy outflow as seismic waves. It assures that the governing system of equations continues to have a solution throughout earthquake-like episodes in which $\partial \delta / \partial t$ increases by several orders of magnitude; the quasi-static model without such a term has solutions in which $\partial \delta / \partial t \rightarrow \infty$ in finite time, with no solution existing beyond that time. Further, the choice $\eta=\mu / 2 c$ both assures that the limiting value of $\partial \delta / \partial t$ is of order $c \Delta \tau / \mu$ (where $\Delta \tau$ is a representative stress drop during an unstable event) and also exactly represents the instantaneous response of continuum elastodynamics along a fault, in the sense of properly relating discontinuities, or sufficiently rapid changes, in the time history of $\tau(x, z, t)$ to corresponding discontinuities, or rapid changes, in the time history of $\partial \delta(x, z, t) / \partial t$. Nevertheless, wave propagation effects along the fault are not modeled by this approximation, and it is important for future study to determine what features of the present results are preserved or modified by rigorous elastodynamic analysis of the propagation and arrest of ruptures.

When slip in the present model is constrained to vary with 


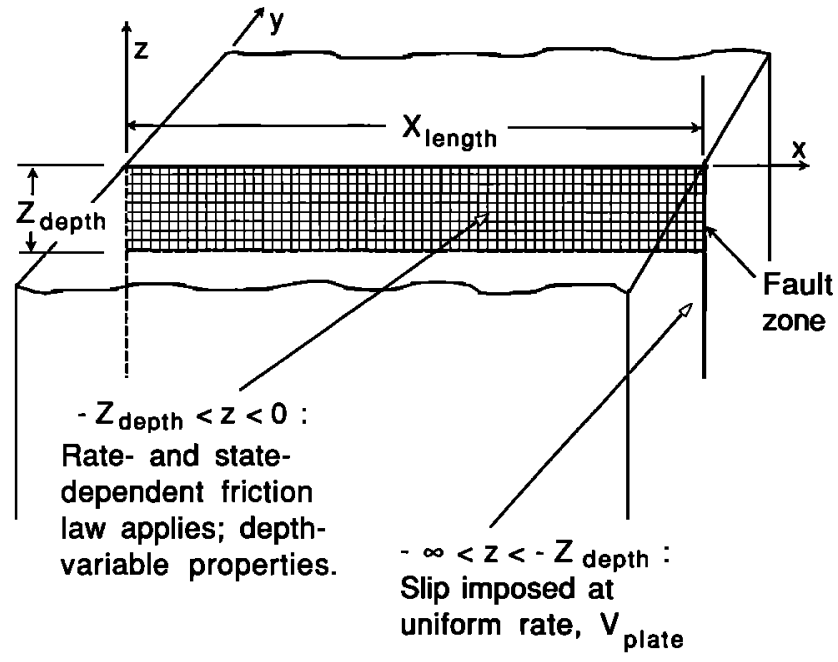

Fig. 1. Vertical fault plane in elastic half-space, driven at uniform rate $V_{\text {plate }}$ below region covered with cells in which rate- and state-dependent friction laws apply. For results reported in subsequent figures, the depth of the zone covered by cells is $Z_{\text {depth }}=24$ $\mathrm{km}$, the periodic repeat distance along strike is $X_{\text {length }}=240 \mathrm{~km}$, and the driving rate is $V_{\text {plate }}=35 \mathrm{~mm} / \mathrm{yr}$.

depth only, $\delta=\delta(z, t)$, so that deformation is by twodimensional antiplane strain, the model coincides approximately with that of Tse and Rice [1986] and makes similar predictions. However, the Tse-Rice analysis gave only limit cycle solutions and did not show the spatio-temporal complexity of the earthquake process, evidenced by the aperiodicity of earthquake occurrence, strong nonuniformities along-strike in the seismic slip distribution, and the Gutenberg-Richter distribution of earthquake sizes. (The Tse-Rice study adopted a different procedure than the inclusion of radiation damping for getting through instability. Instead, when the largest value over the fault of $\partial \delta / \partial t$ in the solution of their quasi-static equations reached a value of order $1 \mathrm{~m} / \mathrm{s}$, they suspended that system of equations in favor of a quasi-static crack analysis to determine the state after instability. The crack analysis solved for the further slip $\delta$ accumulated during instability as the response to a prescribed stress drop $\Delta \tau$ on the rupture, equal to the stress at onset of instability minus the steady state strength for rapid slip; $\Delta \tau$ is negative on hotter, deeper portions of the fault, and the spatial extent of rupture zone was determined as that for which positive and negative stress drops cancelled in the sense of producing no change in stress at the rupture edge. This procedure also allowed them to include dynamic overshoot effects, which made no qualitative change to the results apart from lengthening the interseismic interval. Part of the motivation for using the radiation damping procedure here was to check if the lack of aperiodic solutions found by Tse and Rice could be due to that crack procedure for modeling the instability event.)

In contrast, Horowitz and Ruina [1989] used similar friction laws to those of Tse and Rice [1986] and analyzed sliding between two plates, of finite depth, and of finite width $Y_{b}$ in the $y$ direction, but infinite in $x$ with slip repeated periodically along strike. They drove the system by imposing a uniform fault-parallel displacement rate between the borders at $y= \pm Y_{b}$, assumed completely uniform friction properties over the fault surface, and analyzed the model as a twodimensional elastic problem in the $x-y$ plane, so that slip could vary with distance along strike only, $\delta=\delta(x, t)$. They showed that spatio-temporally complex slip response, while not a generic feature, could result in certain parameter ranges near those of neutral stability for the friction law. They also limited slip velocity runaways with a viscous term.

Thus a first motivation for the present work was to see if the Tse-Rice limit cycle solutions would give way to something more complex if slip was allowed to vary along strike as well as with depth, $\delta=\delta(x, z, t)$, in a system with properties, at each depth, that are uniform or nearly so along strike (but still depth variable because temperature and normal stress vary with depth).

Numerical procedures in the Tse and Rice [1986] and Horowitz and Ruina [1989] studies were devised so that the results had essentially no dependence on such features as the size of cells in a computational grid (Figure 1) or the shortest period considered in a spectral (Fourier series) representation of slip. By choosing such quantities small enough, the solution to the discrete system of numerical equations essentially converged upon the proper continuum limit describing the mathematical model. The same is attempted here in the solutions to be presented. Nevertheless, it seems also useful to focus on the dependence of results, especially for complexity of slip, on cell size since this opens new insights on what may be called "inherently discrete" fault models, by which is meant discrete fault models that have no well-defined continuum limit.

Indeed, the rate- and state-dependent formulation of friction involves a characteristic slip distance $L$ (Figure 2). This is a measure of the distance of sliding necessary for the surface to evolve to a new state, i.e., to a new population of contacting asperities, and can also be interpreted as a sliding distance over which slip weakening takes place. (More accurate descriptions may involve two or more such characteristic distances [Ruina, 1983; Gu et al., 1984]). Requirements for suitable reduction of cell dimension $h$ of a computational grid, or of shortest period in a spectral treatment, scale with $L$. For example, as will be rationalized later, if ( $B$ $-A)_{m}$ represents the maximum value of $\left[-V d \tau^{\mathrm{ss}}(V) / d V\right]$ over the fault plane, where $V$ is local slip rate and $\tau^{\text {ss }}(V)$ is the steady state fictional strength at that rate (Figure 2), then a critical length dimension is defined by $h^{*}=2 \mu L / \pi(B-$ $A)_{m}$. In order for the discrete numerical formulation to coincide with the continuum limit, one requires cell size $h$ $\ll h^{*}$. Subsequent examples will illustrate this point. The oversized cells of an inadequately refined grid can fail independently of one another, whereas the cells of a suitably refined grid are too elastically stiff to slip unstably alone and can do so only as a part of a cooperating group of cells.

Important new perspectives on complexity have been gained in recent studies of discrete fault models. The simplest two-dimensional version of these is a cellular automata [Bak and Tang, 1989; Ito and Matsuzaki, 1990; Chen et al., 1991; Sacks and Rydelek, 1992; Christensen and Olami, 1992; Narkounskaia et al., 1992] with stochastic or uniform deterministic increase in stress on an array of identical cells, or spring-loaded sliding blocks, and with shedding of load to the four nearest-neighbor cells or blocks whenever one fails. These models, which essentially incorporate a simple discontinuous drop from a static to a kinetic friction strength, 
with quasi-static determination of the state after any one cell or block fails, show complex failure sequences described as "self-organized criticality." The frequency of failures involving $N$ cells is found empirically to be an inverse power of $N$ (for $N \gg 1$ but $N$ small compared to overall system size), suggestive of a Gutenberg-Richter frequencymagnitude relationship. Other fault models, with discrete fault patches following static/kinetic friction, and with stress interactions between them calculated by quasi-static elasticity theory, have also shown complex failure sequences [Rundle and Kanamori, 1987; Rundle, 1988a, b; Ward, $1991]$.

The equations of Newtonian dynamics have been solved exactly during slip episodes in other discrete fault models. These have generally involved Burridge and Knopoff [1967] arrays of spring-connected rigid blocks, distributed uniformly in one [Carlson and Langer, 1989; Carlson et al., 1991] or two [Brown et al., 1991; Carlson, 1991] dimensions, with the assumption that friction is a function of velocity only, and is velocity-weakening, once a static threshold force is reached. (Rate- and state-dependent friction, adopted here, reduces to such a description when one lets $L$ $\rightarrow$ 0.) Depending on the ratios of model parameters [Vasconcelos et al., 1992; de Sousa Vieira et al., 1993], the one-dimensional model can also give results suggestive of Gutenberg-Richter behavior for small events. While the equations of Newtonian dynamics are solved exactly in these Burridge-Knopoff models, it has not been generally acknowledged that the dynamical solution for rupture along a chain of lumped masses, or a string of concentrated mass in the continuous limit, bears a presently uncertain relation to dynamical solutions for rupture along a fault embedded in a surrounding elastic continuum. For example, the response of $\mathrm{B}-\mathrm{K}$ models to an instantaneous change in stress $\tau$ along the rupture is an instantaneous change in the acceleration $\partial^{2} \delta / \partial t^{2}$, but there is no instantaneous change in $\partial \delta / \partial t$. By contrast, the response of continuum models is, instead, an instantaneous change in $\partial \delta / \partial t$ (a feature properly modeled by the radiation damping term adopted here). Also, since there is no analogue to energy radiation as seismic waves in the normal implementation of the B-K models (an exception is the recent work of Knopoff et al. [1992]), all potential energy lost to the system during a rupture is fully accountable as frictional work; the same is not true for rupture in a continuum. Related to that point, the B-K model and similar lumped-mass dynamic fault models [e.g., Rice and Tse, 1986] produce large dynamic overshoot of the final slip after rupture, of order $100 \%$ greater than the static slip that would correspond to the stress drop during rupture, and thus leave final stress on any failed block well below that prevailing during unstable sliding. Dynamic overshoot is still not well characterized for rupture in a continuum [Madariaga and Cochard, 1992] but is generally regarded as being far less than that predicted by the lumped-mass fault models. Numerical elastodynamic solutions that prohibit back slip show overshoot of slip on circular ruptures of order $25 \%$ (18-36\% in different simulations), near the center of a rupture, and show smaller overshoot of order 5\% near the edges [Madariaga, 1976; Kostrov and Das, 1988, p. 191].

The cellular automata and Burridge-Knopoff models also simplify elastic interactions to nearest neighbor cells or blocks. Thus they do not properly scale the stress concentration around the rim of a rupture zone with the size of the

\section{Response to a sudden increase in slip speed V}

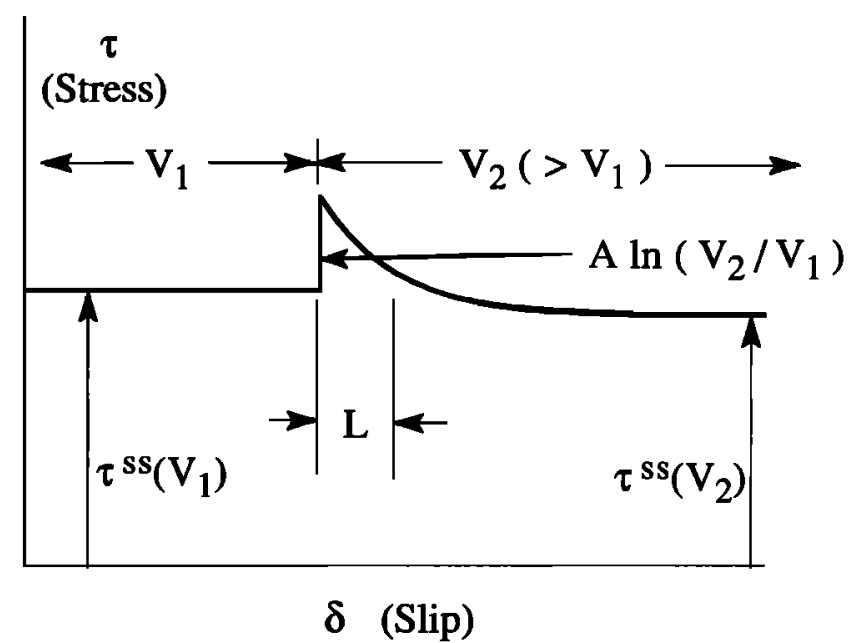

Fig. 2. Features of friction law as revealed by a velocity jump test; $L$ is a characteristic sliding distance associated with evolution of surface state (e.g., formation of a new population of asperity contacts) and slip weakening.

zone and hence do not have the feature that sufficiently large ruptures, growing two dimensionally over a smooth fault plane, will tend to be unstoppable until reaching system boundaries (ruptures that grow large enough two dimensionally to traverse the brittle zone and then spread one dimensionally along strike, in a model like in Figure 1 , do have a stress concentration at their tip which becomes asymptotically independent of rupture area). This feature makes it easier for local heterogeneities of stress to stop ruptures, and those heterogeneities of stress are yet further amplified by the large overshoots in the dynamical B-K models, thus promoting a spectrum of event sizes.

What is focused more on here, however, is that those cellular automata and Burridge-Knopoff models as so far reported are what may be called "inherently discrete" models, in that their simplified friction laws, specifically of a kind that do not include a characteristic slip weakening or state evolution distance $L$, endow them with the property of not having a well-defined continuum limit as the spacing of the discrete elements is shrunk towards zero. Since $L=0$ in those models, $h^{*}=0$ and thus there is no finite characteristic size for the discrete elements to shrink below to pass towards such a limit. (Cao and Aki [1986] also studied a one-dimensional Burridge-Knopoff array, using rate- and state-dependent friction and hence finite $L$, but did not study the statistics of complex slip as systematically as did Carlson and Langer [1989] and Carlson et al. [1991] and, as is relevant here, did not examine the relation of their discrete fault model to its continuum limit.)

The present results, based on solution of the discrete system providing the numerical formulation of the problem sketched in Figure 1, do show richly complex slip when the calculations are done with oversized cells, i.e., of size $h$ that is too large (compared to $h^{*}$ ) to provide an acceptable discretization. Spatio-temporal complexity seems to be generic prediction in that range. Nevertheless, in all cases that have thus far been amenable to study (necessarily with larger $L$ than desirable, for reasons of computational tractability), 
it has been found that spatio-temporal complexity disappears in favor of simple cycles of repeated large earthquakes when cell size of the numerical grid is reduced reasonably below $h^{*}$, i.e., to the range which provides an acceptable discretization of the underlying continuous mathematical model. Thus an emergent view in this field, that spatio-temporally complex slip is a generic feature of mechanical fault models, is contradicted by the present results.

To put a positive interpretation on a seeming defect, however, it may be argued that the oversized cells of an inadequately refined grid might possibly provide a crude first model of a geometrically complex fault zone. Such cells, capable of failing independently of their neighbors, could represent individual fault segments that are poorly connected to adjacent segments, due to bends or offsets, and that can therefore fail independently of one another. Thus a conjecture having consistency with the results found here, but yet to be tested by more rigorous elastodynamic analysis, is as follows: The observed spatio-temporally complex slip in the earthquake process should not be regarded as emerging, at least generically, from nonlinear dynamics acting over an essentially smooth fault plane but rather as emerging as a consequence of geometric disorder of fault zones. That is, natural faults must have characteristic slipweakening distances $L$ associated with them, since such are seen in laboratory studies of friction and faulting. But the influence of geometric disorder over scales in excess of the associated $h^{*}$ causes their large scale dynamics to mimic that of inherently discrete systems, for which spatiotemporal complexity over at least some range of small events seems to be a generic response. This conjecture is based on what could be regarded as artifacts of inadequate numerical discretizations and on calculations over the very limited range of $L$ that is computationally accessible with present algorithms. Nevertheless, it serves to identify a critical open problem of understanding how slip-weakening frictional behavior interacts with fault zone geometric heterogeneity to produce complex earthquake slip.

\section{Modeling Procedures}

Referring to Figure 1, slip is to be calculated on a portion of the fault less deep than $Z_{\text {depth }}$, which includes that portion of the fault which slips in earthquakes. Tectonic loading is represented by imposing uniform motion at the long-term slip rate $V_{\text {plate }}$ on the fault at depths well below those of seismic activity, i.e., at depths greater than $Z_{\text {depth }}$. The fault extends infinitely along strike, but the modeling is done for a slip distribution which is constrained to be repeated periodically along strike with period $X_{\text {length }}$. It is based on equations that embody (1) elasticity of the surroundings and (2) the frictional constitutive relation, in a way now described:

\section{Elastic Relation Between Stress and Slip}

Since the fault plane is embedded in an elastic solid, any distribution of slip motion on the fault will have associated with it a distribution of shearing stress. Thus the right lateral shear stress $\tau(x, z, t)$ along the fault plane, $y=0$, is related to the slip $\delta(x, z, t)$ (constrained to be strike-slip only) by a relation of the type

$$
\tau(x, z, t)=-\int_{0}^{x_{\text {lenglh }}} \int_{-Z_{\text {deph }}}^{0} k\left(x-x^{\prime}, z, z^{\prime}\right)
$$

$$
\cdot\left[\delta\left(x^{\prime}, z^{\prime}, t\right)-V_{\text {plate }} t\right] d x^{\prime} d z^{\prime}-\eta \frac{\partial \delta(x, z, t)}{\partial t}
$$

Related notes are as follows:

1. In terms of the stress and displacement fields, $\sigma_{\alpha \beta}$ and $u_{\alpha}(\alpha, \beta=x, y, z)$, created in the solid, $\tau(x, z, t)=\sigma_{y x}(x$, $0, z, t)$ and $\delta(x, z, t)=u_{x}\left(x, 0^{+}, z, t\right)-u_{x}\left(x, 0^{-}, z, t\right)$.

2. The stiffness kernel $k\left(x-x^{\prime}, z, z^{\prime}\right)$ in the integral is calculated, in discretized form, as the set of stresses at cell centers in Figure 1 from the Chinnery [1963] quasi-static elastic solution for uniform slip over a rectangular dislocation cell in an elastic half space. (T. E. Tullis and W. D. Stuart (private communication, 1992) provided the relevant equations, previously used by Stuart [1986], which were then appropriately summed periodically along strike with period $X_{\text {length }}$; see the appendix.) The integral is thereby discretized into a set of linear relations

$$
\tau_{i}(t)=-\sum_{j} k_{i j}\left(\delta_{j}(t)-V_{\text {plate }} t\right)-\eta d \delta_{i}(t) / d t
$$

where subscripts $i$ or $j$ denote values of variables associated with individual cells, the sum extends over all cells, and the $k_{i j}$ are elements of the stiffness matrix [k] from the Chinnery solution. The diagonal components $k_{\text {diag }}$ of $[k]$ give the relation between slip of a single cell and stress in that same cell. When one omits parts of the expressions for $k_{\text {diag }}$ which remain finite when cell size is reduced towards zero and which arise from the "image" effects of Earth's surface, the remaining part of the expression for $k_{\text {diag }}$ is (as is also derivable from expressions for stresses due to dislocations in full spaces [e.g., Hirth and Lothe, 1968])

$$
k_{\text {diag }}=\frac{2 \mu}{\pi h} \frac{1+q^{2} /(1-\nu)}{\left(1+q^{2}\right)^{1 / 2}}
$$

where $h$ is the edge length of a single cell of Figure 1 in the depth direction, $h / q$ is the edge length in the along-strike direction, $\mu$ is the elastic shear modulus, and $\nu$ is the Poisson ratio. The expression for $k_{\text {diag }}$ is used to extract the critical cell dimension $h^{*}$.

3. The factor $\eta$ describes seismic radiation damping, adopted to avoid the unbounded slip velocity which would otherwise develop as a consequence of instability in a quasi-static model, but to do so in a more tractable way than would be the case if the entire calculation were set up in the (proper, and preferable) elastodynamic framework. The most sensible choice of $\eta$ is $\mu / 2 c$, where $c$ is shear wave speed. This describes the relation between stress and slip rate for spatially uniform but arbitrarily time-dependent slip $\delta=\delta(t)$ along an infinite fault plane $y=0$ traversing an elastic full space, under conditions that all radiation is as outgoing planar shear waves. The result may be seen as follows: For such a wave field, $\sigma_{y x}+(\mu / c) \partial u_{x} / \partial t$ is constant in space and time in $y>0$, just as $\sigma_{y x}-$ $(\mu / c) \partial u_{x} / \partial t$ is in $y<0$; on $y=0, \sigma_{y x}^{+}=\sigma_{y x}^{-}=\tau$ and $\delta=$ $u_{x}^{+}-u_{x}^{-}$, so that $\tau=$ const $-(\mu / 2 c) \partial \delta / \partial t$ on the fault, showing the origin of $\eta=\mu / 2 \mathrm{c}$. In fact, with this value for $\eta$ the above equation for $\tau(x, z, t)$ along the fault plane, or for $\tau_{i}(t)$ in the discretized form, exactly incorporates the elastodynamic result for how any instantaneous changes in the time histories of $\tau(x, z, t)$ and $\partial \delta(x, z, t) / \partial t$, or of $\tau_{i}(t)$ and $d \delta_{i}(t) / d t$, are related to one another. It is clear that if the 
constitutive response of the fault zone material causes a stress $\tau$ to decrease by a stress drop $\Delta \tau$ over a time scale for which the slips $\{\delta\}$ change only very modestly, so that elements of the product $[k]\{\delta\}$ changes little in comparison to $\Delta \tau$, then the resulting slip velocity $d \delta / d t$ will be of order $\Delta \pi / \eta$, i.e., of order $c \Delta \tau / \mu$, which is the proper range for slip rates during an inertia limited instability in an elastic continuum. The exact elastodynamic equations would, however, replace the product $[k]\{\delta\}$ with a convolution in time, and that convolution reduces to the quasi-static product form used here only on time scales that are large compared to those for waves to travel representative length scales along the fault over which there are gradients in the slip distribution. Thus, as suggested earlier, important aspects of the elastodynamics of fault response, especially relating to wave-mediated propagation along the fault plane of stress alterations due to slip, are not adequately treated here. Instead, stress changes corresponding to those predicted by an exact elastodynamic analysis after wave propagation (i.e., the final static stress changes) are communicated instantaneously along the fault by the system of equations adopted here.

4. While $\mu / 2 c$ is the proper choice for $\eta$ in the present formulation, large values of $\eta$ shorten computer time required to integrate through instabilities; thus $\eta=10^{2}$ to $10^{4}$ $\mu / 2 c$ is typically used and sometimes $10^{6} \mu / 2 c$. This can be reinterpreted as doing the analysis with a much lower wave speed, say, $\hat{c}$, than actual speed $c$, but one which, for the parameters adopted, still assures that the typical unstable slip rate $\hat{c} \Delta \tau / \mu$ is many orders of magnitude larger than the loading rate $V_{\text {plate }}$ or interseismic slip rates. The use of such large $\eta$ values has the effect of extending the time of individual instabilities to time scales of minutes to days. Since the higher $\eta$ is, the lower the maximum slip velocity attained during instability is, there is another effect for the type of friction law used here, with monotonically decreasing $\tau^{\text {ss }}(V)$ : Higher $\eta$ means lower stress drop, and hence slip, in individual earthquakes and thus a shorter recurrence interval. (This would be a small or negligible effect in one version of the friction laws used by Tse and Rice [1986] which had a "leveling off" of $\tau^{\text {ss }}(V)$ at slip speeds above about $1 \mathrm{~mm} / \mathrm{s}$.) For example, in a test case involving periodically repeated earthquakes, a recurrence interval of 90 years obtained with $\eta=\mu / 2 c$ shortened to 74 years with $\eta=$ $10^{2} \mu / 2 c$, to 55 years with $\eta=10^{4} \mu / 2 c$, and to 35 years with $\eta=10^{6} \mu / 2 c$.

5. In the above equations for stress $\tau$ in terms of slip $\delta$, it may at first seem unclear why the integration extends over only the part $-Z_{\text {depth }}<z<0$ of the half plane and not below where slip $\delta=V_{\text {plate }} t$ is imposed. The logic for this may be seen by decomposing the slip $\delta$ into the sum of a part $V_{\text {plate }} t$ that is uniform over the entire half plane plus a part $\delta-$ $V_{\text {plate }} t$ which is nonuniform. The former is associated with a rigid translation of one elastic quarter space relative to the other and creates no stress; the latter is nonzero only over $-Z_{\text {depth }}<z<0$, which is therefore the source region in the integral or sum over cells. (See also Rundle $[1988 a, b]$.)

\section{Frictional Constitutive Relation}

The most common rate- and state-dependent friction law of the class introduced by Dieterich [1981] and Ruina [1983] is used and can be written

$$
\begin{gathered}
\frac{\partial \tau}{\partial t}=\frac{A}{V} \frac{\partial V}{\partial t}-\frac{V}{L}\left[\tau-\tau^{\mathrm{ss}}(V)\right] \\
V(x, z, t)=\frac{\partial \delta(x, z, t)}{\partial t}
\end{gathered}
$$

where here $\boldsymbol{A}(>0)$ is taken as a constant, i.e., independent of $V$ and $\tau$ but not of effective normal stress and temperature. Also, the form adopted for the steady state strength is

$$
\tau^{\mathrm{ss}}(V)=\tau_{0}+(A-B) \ln \left(V / V_{\text {plate }}\right),
$$

where $\tau_{0}, A-B$, and $L$ are constant in the same sense. This is the law used by Tse and Rice [1986] and is commonly seen in an equivalent version [Ruina, 1983], which explicitly introduces an evolving state variable $\theta$, as the set of equations

$$
\begin{gathered}
\tau=\tau_{0}+A \ln \left(V / V_{\text {plate }}\right)+B \theta \\
\partial \theta / \partial t=-(V / L)\left[\theta+\ln \left(V / V_{\text {plate }}\right)\right]
\end{gathered}
$$

The logarithmic forms give approximate fits to data over, typically, a few orders of magnitude variation in sliding rate; low or high speed cutoffs are sometimes introduced. The main features of this friction law can be seen from the integration of the equations defining it for the idealized velocity jump test in Figure 2. In this test an imposed slip rate is suddenly changed from a steady value $V_{1}$ to a new value $V_{2}$; the resulting stress versus slip is shown. For sustained slip $\delta \gg L$ at fixed $V\left(=V_{1}\right.$ or $\left.V_{2}\right)$ the response is "steady state" friction, and it is appropriate to adopt the simplified viewpoint that $\tau$ is just a function of $V$, namely, $\tau$ $\approx \tau^{s s}(V)$. This corresponds to (steady state) velocity strengthening if $\boldsymbol{A}-\boldsymbol{B}>\mathbf{0}$, and to weakening if $\boldsymbol{A}-\boldsymbol{B}<$ 0 . However, when the sliding velocity is suddenly increased or decreased over amounts of slip that are small compared to $L$, one finds that the stress respectively increases or decreases. This is a fundamental feature of material response, not an inertial effect in the testing apparatus. With $A \equiv V$

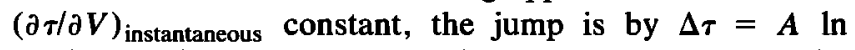
$\left(V_{2} / V_{1}\right)$ as in Figure 2. According to the law adopted, the approach to a new steady state involves exponential decay such that if slip $\delta=V_{2} t$ is measured from the moment of the jump, then $\tau$ evolves toward the new steady state strength level $\tau^{s s}\left(V_{2}\right)$ according to the relation

$$
\tau=\tau^{\mathrm{ss}}\left(V_{2}\right)+e^{-\delta / L}\left[\tau^{\mathrm{ss}}\left(V_{1}\right)+A \ln \left(V_{2} / V_{1}\right)-\tau^{\mathrm{ss}}\left(V_{2}\right)\right]
$$

The bracketed term represents the drop in strength, or slip weakening, from the peak strength $\tau^{\mathrm{ss}}\left(V_{1}\right)+A \ln \left(V_{2} / V_{1}\right)$, required to initiate slip at the rapid rate $V_{2}$, to the residual level $\tau^{\text {ss }}\left(V_{2}\right)$ attained after slip $\delta \gg L$. Thus in different limiting situations the rate- and state-dependent friction formulation includes not only velocity weakening but also slip-weakening type behavior. The latter is equivalent to the notion of fracture energy at crack tips in singular elastic crack theory [Rice, 1980], and the fracture energy for the above idealized representation of the stress versus slip relation is just the bracketed term times $L$. (This description is idealized because $V$ is highly variable rather than uniform at some large value $V_{2}$ behind the front of an advancing slip zone, and steady state slip conditions at some low slip rate $V_{1}$ will not generally prevail ahead of the front.) Descriptions of fault friction that do not include some such charac- 

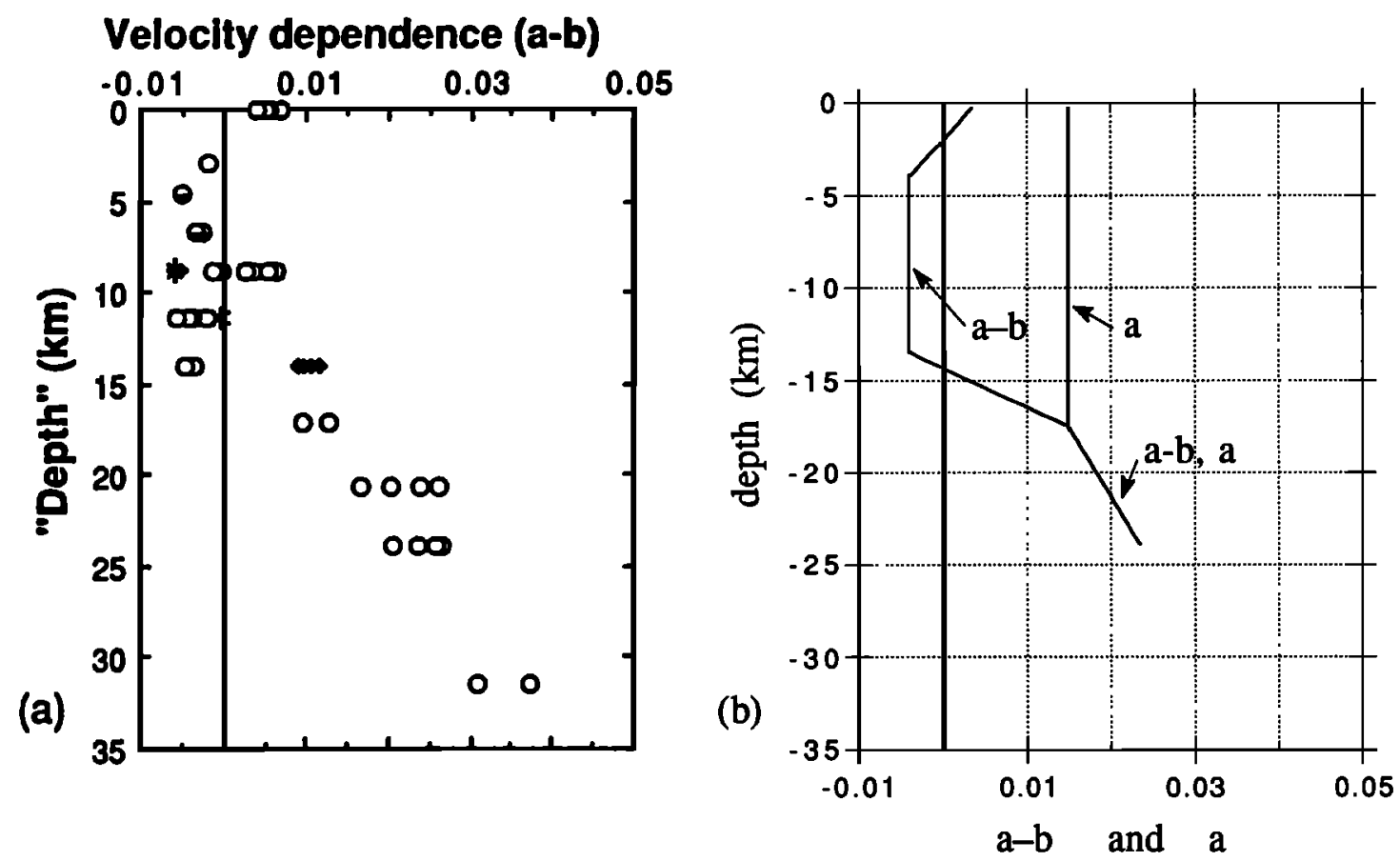

Fig. 3. (Left) Laboratory data, granite under hydrothermal conditions, on velocity weakening/strengthening at various temperatures; from Blanpied et al. [1991] as converted by them into $a-b\left[=V d \tau^{\mathrm{ss}}(V) / d V /\left(\sigma_{n}-p\right)\right]$ versus depth based on a Lachenbruch-Sass SAF geotherm. (Right) Assumed $a-b$ and $a\left[=V(\partial \tau / \partial V)_{\text {instantaneous }} /\left(\sigma_{n}-p\right)\right]$ versus depth for use in model of Figure 1.

teristic length $L$ are then equivalent to degenerate elastic crack models with zero fracture energy. In such models, cracks in an elastic continuum can only propagate at wave speeds (for example, an antiplane shear crack at the shear wave speed, an in-plane shear crack at the Rayleigh speed) and cannot concentrate stress ahead of their tips; any nonzero stress intensity factor, as is generally associated with subsonically propagating cracks, corresponds to a positive fracture energy.

In formulating the friction law for the model of Figure 1 the following assumptions are adopted:

1. The coefficients $A$ and $B$ scale directly with effective normal stress, $A=a\left(\sigma_{n}-p\right)$ and $B=b\left(\sigma_{n}-p\right)$, where $\sigma_{n}\left(=-\sigma_{y y}\right)$ is the compressive normal stress on the fault and $p$ is pore pressure, and the coefficients $a$ and $b$ then depend only on temperature.

2. The velocity dependence $a-b$ is made depth variable following the data of Blanpied et al. [1991] on temperature dependence of $a-b$ for granite under hydrothermal conditions. They converted their data on $a-b$ as a function of temperature to a plot of $a-b$ as a function of depth, as based on a Lachenbruch-Sass San Andreas fault (SAF) geotherm, and their plot is shown in Figure 3 at the left. These data show transition from velocity weakening, $a-b$ $<0$, to velocity strengthening, $a-b>0$, at temperatures around $325^{\circ} \mathrm{C}$, corresponding to depth $z \approx-14 \mathrm{~km}$. There is also a velocity strengthening zone at shallow depth, above $-2 \mathrm{~km}$. The fit to $a-b$ and the values of $a$ used in the modeling are shown in Figure 3 at the right; $a-b$ has a minimum value of -0.004 over depths from 4.0 to $13.5 \mathrm{~km}$ and is negative from 2.0 to $14.3 \mathrm{~km}$; $a$ is not reported by Blanpied et al. [1991] but factor of 2 or so variations in its value, for a given distribution of $a-b$, have little effect on the dynamics.

3. The normal stress $\sigma_{n}=(280 \mathrm{bar} / \mathrm{km})(-z)[(28$ $\mathrm{MPa} / \mathrm{km})(-z)]$, a typical overburden stress, and the pore pressure $p$ is given by either $p=$ hydrostatic $=(100 \mathrm{bar} / \mathrm{km})$ $(-z)[(10 \mathrm{MPa} / \mathrm{km})(-z)]$, or by $p=\max$ (hydrostatic, $\sigma_{n}-$ const). The latter incorporates elevated pore pressure concepts [Rice, 1992] in which $d p / d z$ approaches $d \sigma_{n} / d z$ with increase of depth, as a consequence of slow upward fluid permeation in a fault with permeability that diminishes rapidly with increase of $\sigma_{n}-p$; see Figure 4, which illustrates this type of pressure distribution for $p$ offset by 1000 bars (100 MPa) from $\sigma_{n}$ at depth.

4. The slip weakening, or state transition, slip distance $L$ is in the $0.01-$ to $0.1-\mathrm{mm}$ range in data involving small slips on laboratory-prepared fault surfaces that are smooth at wavelengths above a $\mathrm{mm}$ or so [Dieterich, 1981; Biegel et al., 1989]. Slip weakening distances that are typically in the range $\mathbf{0 . 1}-0.3 \mathrm{~mm}$ occur in fault formation in initially intact laboratory triaxial specimens of granite [Rice, 1980; Wong, 1982]. It is currently a computational necessity to make $L$ larger, above $2 \mathrm{~mm}$ in the 2-D modeling and above $10 \mathrm{~mm}$ in the 3-D modeling, to accurately solve equations with suitably refined grid sizes to reach the continuum limit. Larger $L$ values may also be partially justified to approximately accommodate scaling to broad scale fault roughness (Scholz [1988] suggests $L \approx 1$ to $10 \mathrm{~mm}$ over a $6-16 \mathrm{~km}$ depth range based on asperity contacts along fractal fault surfaces) and to model weakening in large rapid slip for which constraints from the fracture energy of earthquakes $\left(G=10^{6}\right.$ to $10^{7}$ $\mathrm{J} / \mathrm{m}^{2}$, strength drops in rupture $=2 \times 10^{2}$ to $10^{3}$ bars $[20-100$ $\mathrm{MPa}$ ], $L \approx G /$ strength drop) suggest a 10 - to $500-\mathrm{mm}$ range. 
Normal stress ( $\sigma_{n}$ ) and pore pressure ( $p$ ) versus depth

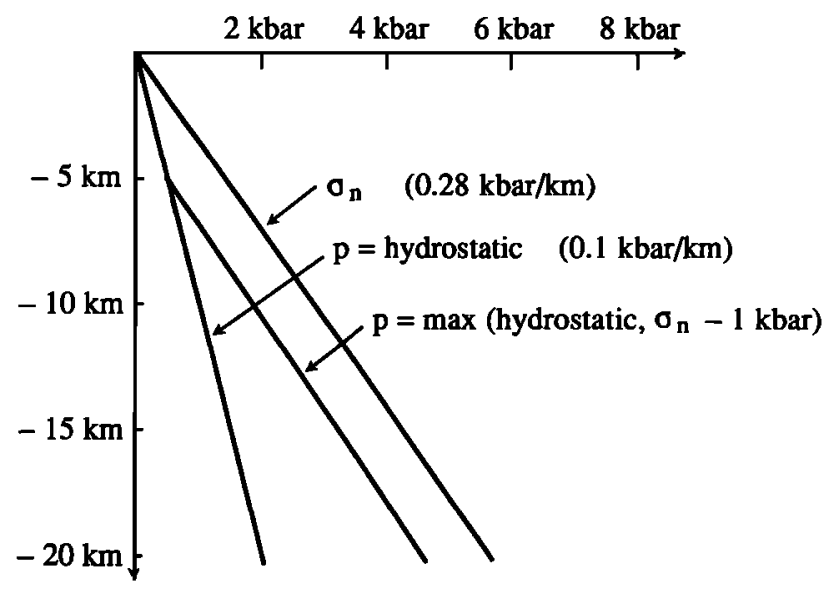

Fig. 4. Forms assumed for pore pressure versus depth. Pore pressure $p$ may be hydrostatic or may have a transition to a rate of increase with depth that is the same as for normal stress $\sigma_{n}$; the latter case approximately represents results of Rice [1992] for slow pore fluid upflow along a fault zone with permeability that diminishes rapidly with increase of $\sigma_{n}-p$. Case shown is for $p=\max$ (hydrostatic, $\sigma_{n}-1000$ bars [100 MPa]).

It seems possible that $L$ values in the same range as inferred for small slips in laboratory specimens will apply to the onset of slip on natural faults but that the far larger slips of such earthquakes involves a much larger scale slip-weakening length in subsequent slip. This might be describable approximately by the multi-state-variable form discussed by Ruina [1983] and used by $G u$ et al. [1984] in stability analysis, but the simplification of a single slip-weakening process is retained in the present work.

5. The constant term in the friction law can be written as $\tau_{0}=f_{0}\left(\sigma_{n}-p\right)$, where $f_{0}$ is the friction coefficient for steady state sliding at the rate $V=V_{\text {plate. For a given }}$ distribution of $A$ and $B$ over the fault plane, the term $\tau_{0}$ is irrelevant for the results obtained for the slip rate $V(x, z, t)$ once the system has gone through an early part of its history, sensitive to initial conditions.

Thus if we introduce the notation $s_{i}=\tau_{i}-\tau_{i}^{\mathrm{ss}}\left(V_{i}\right)$, where subscript $i$ refers to the value of a variable, or the form of a function like $\tau_{i}^{\mathrm{ss}}(V)$ or $d \tau_{i}^{\mathrm{ss}}(V) / d V$, in cell $i$ of the grid, the governing equations are

$$
\begin{gathered}
\left(\eta+A_{i} / V_{i}\right) \dot{V}_{i}=-\sum_{j} k_{i j}\left(V_{j}-V_{\text {plate }}\right)+V_{i} s_{i} / L \\
\left(\eta+A_{i} / V_{i}\right) \dot{s}_{i}=-\left[A_{i} / V_{i}-d \tau_{i}^{\mathrm{ss}}\left(V_{i}\right) d V_{i}\right] \\
\cdot \sum_{j} k_{i j}\left(V_{j}-V_{\text {plate }}\right)-\left[\eta+d \tau_{i}^{\mathrm{ss}}\left(V_{i}\right) / d V_{i}\right] V_{i} s_{i} / L
\end{gathered}
$$

where the overdots denote a time rate. We note that $\eta$ is always much less than $A_{i} / V_{i}$ and $\left|d \tau_{i}^{\text {ss }}\left(V_{i}\right) / d V_{i}\right|\left(=\mid A_{i}-\right.$ $B_{i} \mid / V_{i}$ ) when $V_{i}$ is of the order of $V_{\text {plate }}$ but that $\eta$ dominates those terms for the large $V_{i}$ which result during an earthquake instability. Because of the "stiffness" of this set of differential equations, an implicit integration procedure has been devised and is explained in the appendix.

In all cases discussed here the parameters $Z_{\text {depth }}=24 \mathrm{~km}$,
$X_{\text {length }}=240 \mathrm{~km}, V_{\text {plate }}=35 \mathrm{~mm} / \mathrm{yr}, \mu=300 \mathrm{kbar}(30 \mathrm{GPa})$, $\nu=0.25$ and $c=3 \mathrm{~km} / \mathrm{s}$ are used. Thus the variables whose effects are illustrated here are $L$, the "constant" in $p=\max$ (hydrostatic, $\sigma_{n}-$ const), and the radiation damping $\eta$, as well as variables defining the computational grid. In principle, the latter should not be regarded as variables, in that one assumes that cell size $h$ is taken small enough to be irrelevant to the results shown. However, as mentioned in the introduction, possible insights on the origin of spatiotemporal complexity of slip are obtained by examining how solutions depend on $h$.

The grid involves a number $N_{\text {depth }}$ of identical cells in any single column through the $24 \mathrm{~km}$ depth range and $N_{\text {length }}$ columns of such cells along the $240-\mathrm{km}$ periodic repeat distance along strike. The height of a single cell is thus $h=$ $24 \mathrm{~km} / N_{\text {depth. }}$. In the 3-D results to be presented, the cells have $q=0.8$ which assures similar contributions (within $7 \%$ ) to $k_{\text {diag }}$ as expressed above from terms representing screw and edge dislocation segments along a cell border. Since $h / q=240 \mathrm{~km} / N_{\text {length }}$ this means that $N_{\text {length }}$ is always taken as $8 N_{\text {depth }}$ in the 3-D examples. For the 2-D version of the model, approximately comparable to that of Tse and Rice [1986], $X_{\text {length }}$ is unbounded and $N_{\text {length }}=1$, corresponding to $q=0$. The (dominant part of the) single cell stiffness is then $k_{\text {diag }}=1.45(2 \mu / \pi h)$ for the 3-D cases, where $k_{\text {diag }}=2 \mu / \pi h$ is the stiffness for an infinitely long cell in the $x$ direction corresponding to the 2-D version of the model.

To estimate a critical cell size $h^{*}$, above which cells are capable of failing independently of one another, note that a critical spring stiffness for a single-degree-of-freedom system following the friction law adopted is $k_{\text {crit }}=(B-A) / L$; here $k$ is the reduction in shear stress for unit slip on the sliding surface due to elastic interactions with the surroundings. This was shown by Ruina [1983] and Rice and Ruina [1983] to be the critical stiffness according to linear stability theory for a simple spring-slider system, in that perturbations from steady state slip in a system with steady state velocity weakening ( $B-A>0$ ) will grow in time only at lesser spring stiffness. Nonlinear stability studies based on that law [Gu et al., 1984] show that for $k$ above $k_{\text {crit }}$ instability can result only if the system is finitely disturbed (e.g., by a sudden change in stress or loading rate), with the amplitude of the disturbance in loading rate that is required for instability growing exponentially with $k / k_{\text {crit }}$. Thus, to assure that the mesh is not so coarse that individual cells can act independently of one another, we need to make the selfstiffness of a single cell, which can be written approximately as $k_{\text {diag }}=2 \mu / \pi h$, large compared to $k_{\text {crit }}$. Defining $h^{*}$ as the grid size that makes $k_{\text {diag }}=k_{\text {crit }}$ for the most critical (highest $B-A)$ cell in the grid, this gives $2 \mu / \pi h^{*}=(B-A)_{m} / L$, and thus

$$
h^{*}=2 \mu L / \pi(B-A)_{m}
$$

as earlier. Hence the requirement $k_{\text {diag }} \gg k_{\text {crit }}$ for a suitable grid translates into the requirement $h \ll h^{*}$. The size $h^{*}$ may be called the nucleation size, in that it measures approximately the minimum diameter of a patch of fault area which must be able to slip simultaneously in order for an instability to develop. When discussing computational results next, the ratio $h / h^{*}$ for the grid on which they are based is given in each case. 


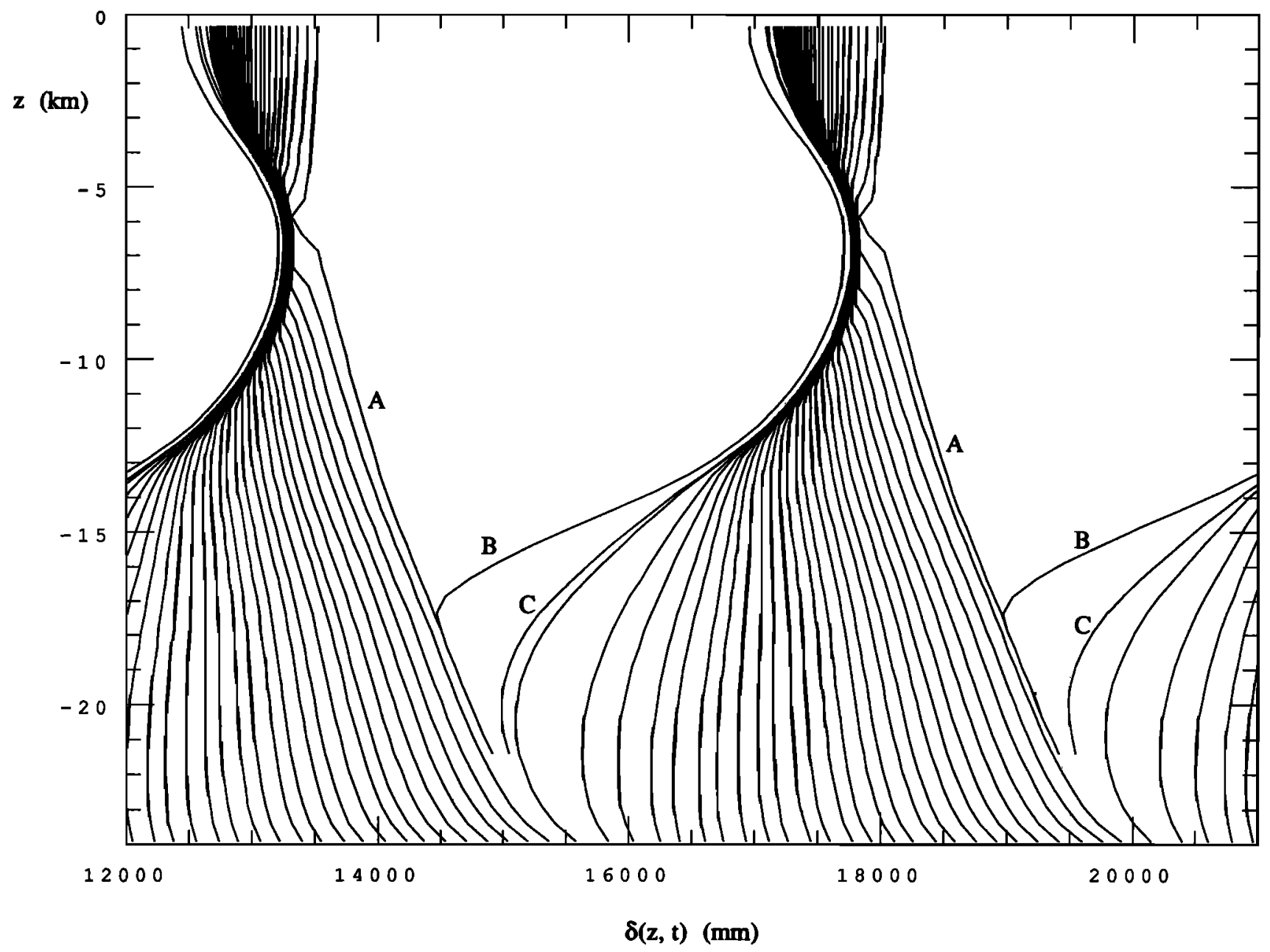

Fig. 5. Slip in millimeters on horizontal axis versus depth in kilometers on vertical axis, drawn through the full depth range every 5 years and drawn through partial depth range to mark special features: $A$, onset of instability (maximum velocity among cells increases through $10^{3} V_{\text {plate }}$ ); B, one week after instability; and $C$, one year after instability. For 2-D version of model in Figure 1, slip constrained to be the same at every position along strike [ $\delta=\delta(z$, $t$ )], $L=30 \mathrm{~mm}, p=\max \left(\right.$ hydrostatic, $\sigma_{n}-1000$ bars $\left.[100 \mathrm{MPa}]\right), \eta=10^{2} \mu / 2 c$. For 96 cells over $24 \mathrm{~km}$ depth $\left(h / h^{*}\right.$ $=0.17)$.

\section{SOME RESULTS}

Two-Dimensional Cases $\delta=\delta(z, t)$

As in the work by Tse and Rice [1986], in every model studied so far with adequate reduction of cell size, a simple limit cycle solution of periodic large earthquakes occurs.

Figure 5 shows slip versus depth for a case with $L=30$ $\mathrm{mm}, p$ offset from $\sigma_{n}$ by 1000 bars $(100 \mathrm{MPa})$ at depth (Figure 4), and $\eta=10^{2} \mu / 2 c$. Since $(B-A)_{m}=(0.004) \times$ $(1000$ bars $)=4$ bars $(0.4 \mathrm{MPa})$, this case has $h^{*} \approx 1.5 \mathrm{~km}$. The calculation was done with 96 cells over the $24 \mathrm{~km}$ depth range; hence $h=0.25 \mathrm{~km}$ and $h / h^{*} \approx 0.17$, a ratio small enough to reasonably represent the continuum limit. The slip distribution every fifth year is shown by curves running from top to bottom in Figure 5; those that are truncated before reaching bottom show the slip distribution at the onset of instability (A), and then one week (B) and one year (C) later. There is rapid postseismic slip at depth.

Figures 6 and 7 show results for a much smaller $L$ value, $L=3 \mathrm{~mm}$, and a more highly elevated pore pressure, offset from $\sigma_{n}$ by 500 bars $(50 \mathrm{MPa})$ at depth, so that $h^{*} \approx 0.3 \mathrm{~km}$, and for $\eta=10^{4} \mu / 2 c$. Each of the figures has two panels, top one showing results for an inadequately refined grid ( 32 cells, $h / h^{*} \approx 2.5$ ), and bottom one for a refined grid ( 320 cells, $h / h^{*} \approx 0.25$ ) that is in the range of approach to the continuum limit. Figure 6 shows slip versus depth every year. Figure 7 shows, as a function of time, the logarithm of the slip rate in whichever is the fastest slipping cell at the time of a sample taken every 0.01 year (the fastest slip rates tend not to show since they form spikes narrower than $\mathbf{0 . 0 1}$ year). These results are typical for 2-D cases in showing how the spatio-temporally complex slip predicted for oversized cells gives way to simple limit cycles with reduction of cell size to ranges corresponding to approach to the continuum limit. The many spikes in the top panel of Figure 7, and the ragged accumulated slip profiles in the top panel of Figure 6, correspond to a complex failure mode with many small instabilities in a large earthquake cycle and aperiodic occurrence of the large events. These features all disappear with grid reduction and thus are artifacts of an inadequate numerical scheme. Possibly, they may also hint at the way that geometric disorder controls the dynamics of fault zones.

Comparing Figure 5 to the bottom panel of Figure 6, the results show how precursory slip effects that appear in 

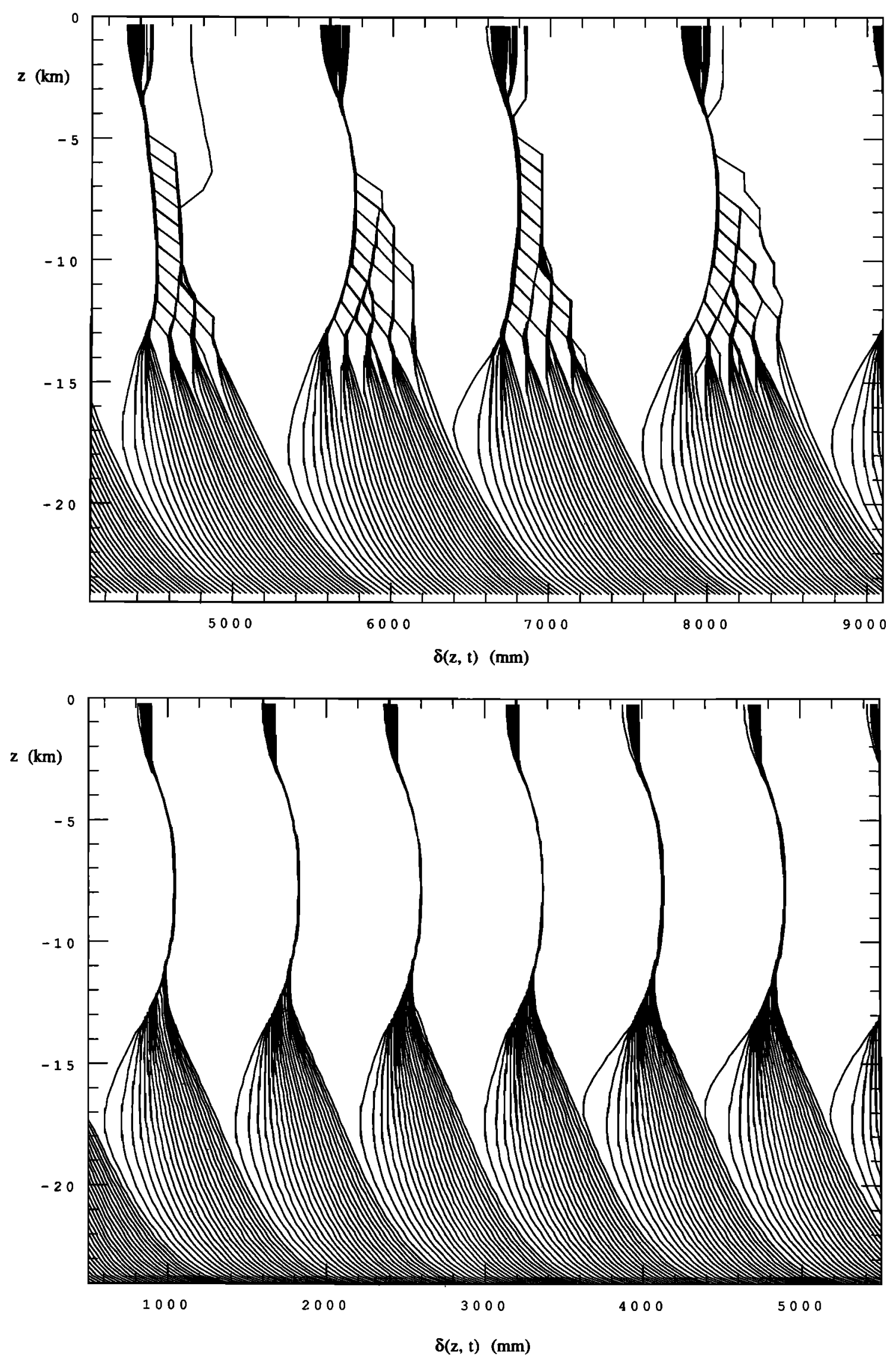

Fig. 6. Slip in millimeters on horizontal axis versus depth in kilometers on vertical axis, drawn every 1 year. For 2-D version of model in Figure 1 with slip constrained to be the same at every position along strike $[\delta=\delta(z, t)]$, and for $L=3 \mathrm{~mm}, p=\max$ (hydrostatic, $\sigma_{n}-500$ bars [50 MPa]), $\eta=10^{4} \mu / 2 c$. (Top) For 32 cells over $24 \mathrm{~km}$ depth $\left(h / h^{*}=2.5\right)$. (Bottom) For 320 cells over $24 \mathrm{~km}$ depth $\left(h / h^{*}=0.25\right)$. 

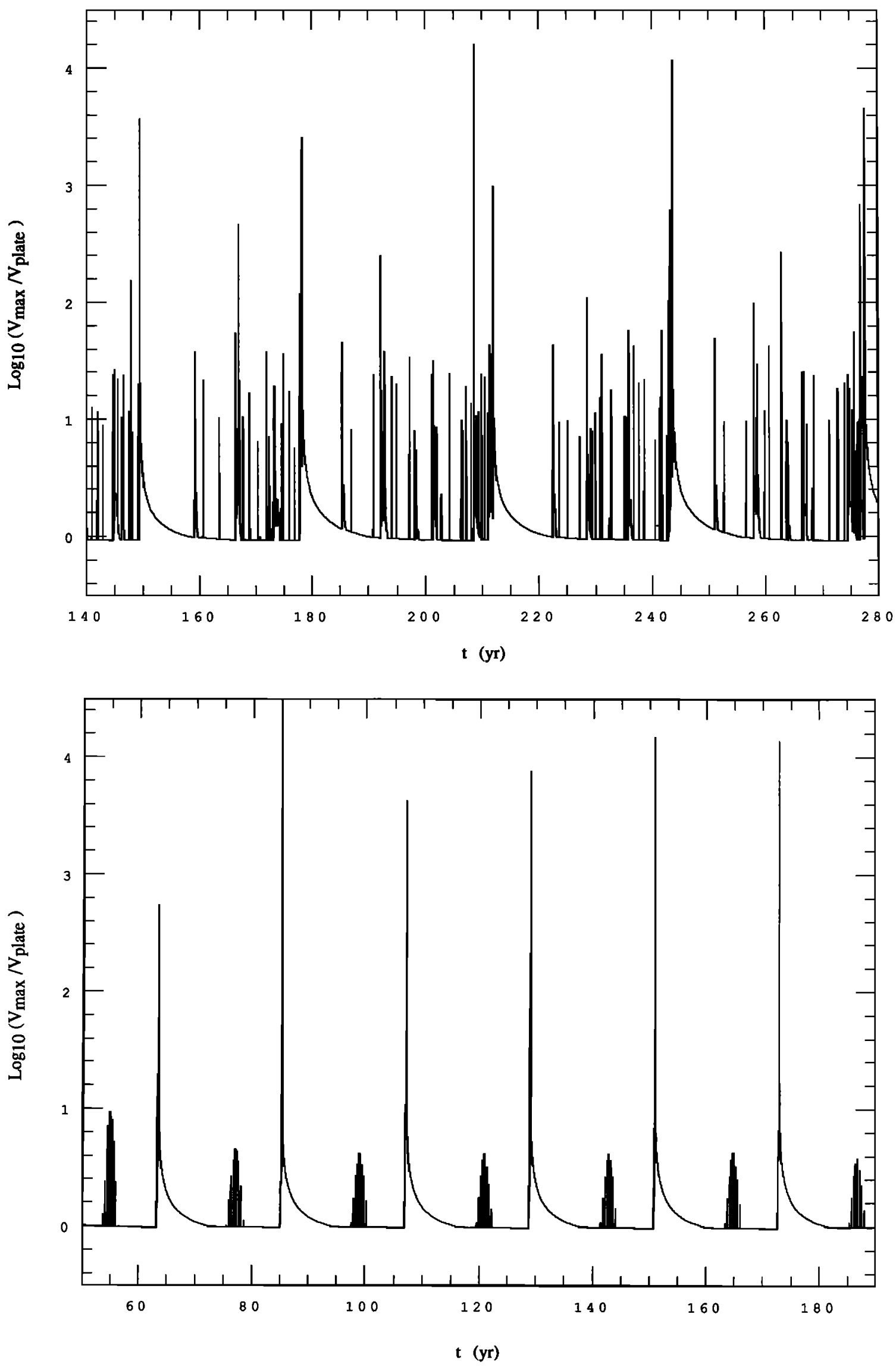

Fig. 7. Maximum value over all cells of $\log _{10}\left\{[\partial \delta(z, t) / \partial t] / V_{\text {plate }}\right\}$, as sampled every 0.01 year, on vertical axis versus time in years on horizontal axis; parameters as in Figure 6. (Top) For 32 cells over $24 \mathrm{~km}$ depth $\left(h / h^{*}=2.5\right)$. (Bottom) For 320 cells over $24 \mathrm{~km}$ depth $\left(h / h^{*}=0.25\right)$. 
simulations with large $L$ diminish to essentially imperceptible size with small $L$; the comparison here is $L=30 \mathrm{~mm}$ and $L=3 \mathrm{~mm}$, and the latter is still a factor of 100 or so larger than $L$ for laboratory fault surfaces that have been machined to be smooth at large spatial wavelengths. Unfortunately, with present algorithms (the appendix), which are efficient between earthquakes but require much time to integrate through earthquake instabilities, the 320 cell 2-D case required 5 days cpu time on a Sparcstation 2 to cover about 210 years of imposed motion, or about $7.3 \mathrm{~m}$ total slip travel. During that time, eight earthquakes occurred; the first, influenced by starting conditions, after 42 years, and the rest with a cycle time of about 22 years. The limit cycle solution illustrated in the bottom panels of Figures 6 and 7 was essentially established by the time of the second earthquake.

\section{Three-Dimensional Cases $\delta=\delta(x, z, t)$}

The 3-D cases are yet more restricted by computational limits. With present algorithms, a grid with 32 cells through the thickness, and thus 256 columns of cells along strike, is the most refined possible, and even then 13 days of Sparcstation $2 \mathrm{cpu}$ time were required for a run of 300 years in one of the easiest cases with large $L(40 \mathrm{~mm})$ and large $\eta\left(10^{6}\right.$ $\mu / 2 c)$. Large $L$ increases the time step size allowed; large $\eta$ decreases the number of time steps required to integrate through instabilities, which more than pays for its subsidiary effect of reducing stress drop and recurrence intervals and hence increasing the number of large events over some long time span. Grids of that size, $N_{\text {length }}=256$ and $N_{\text {depth }}=32$, are possible at all, at least within the present algorithm, only because fast Fourier transform (FFT) techniques can be used to do the matrix multiplications involving $[k]$ that are required in each time step; see the appendix. Because of the FFT involvement, in the simple form adopted based on powers of 2 , the next smaller grid has 128 cell columns along strike and hence only 16 cells through the thickness (to maintain the cell aspect ratio).

In order to rapidly bring to evidence any tendencies for instability in the 3-D modeling, the constitutive properties $a$ and $a-b$ were perturbed as follows. Starting with these variables as a function of depth as in Figure 3 at the right, the value at each depth was reduced by $10 \%$ in the first $60 \mathrm{~km}$ along strike, left unaltered over the next $120 \mathrm{~km}$, and reduced by $5 \%$ over the final $60 \mathrm{~km}$ of a $240-\mathrm{km}$ period.

Figure 8 shows results for a case with $L=40 \mathrm{~mm}$ and hydrostatic pore pressure, so that $h^{*} \approx 0.79 \mathrm{~km}$, and with $\eta$ $=10^{6} \mu / 2 c$. Again, two panels are shown, the top one for a coarse grid of 2048 cells $\left(N_{\text {length }}=128, N_{\text {depth }}=16\right)$ with $h / h^{*}=1.9$, and the bottom one for a more refined grid of 8192 cells $\left(N_{\text {length }}=256, N_{\text {depth }}=32\right)$ with $h / h^{*}=0.95$. In this case the slip averaged over $24 \mathrm{~km}$ depth,

$$
\bar{\delta}(x, t)=\frac{1}{Z_{\text {depth }}} \int_{-Z_{\text {deph }}}^{0} \delta(x, z, t) d z,
$$

is shown, at 5-year intervals, as a function of distance $x$ along strike over one $240-\mathrm{km}$ period. Results are shown from the last 300 years of an 800-year calculation. The coarse grid results show a complex history with strongly locked zones forming and rupturing a few times over but then transitioning to failure in events with less slip while strong locking regions form elsewhere. While for a grid that is still far from a ratio $h / h^{*}$ that is convincingly at the continuum limit, the refined grid results, have essentially lost all of this complexity.

Figures 9 and 10 compare slip versus depth, printed at 5-year intervals, for these two cases. Figure 9 shows the slip profiles at $x=0.25 X_{\text {length }}=60 \mathrm{~km}$ and Figure 10 for $x=$ $0.75 X_{\text {length }}=180 \mathrm{~km}$; in each case the top panel is for the coarse grid and the bottom is for the more refined grid. Comparing the top panels in Figures 9 and 10, one sees a chaotic slip history with qualitative changes in the fault locking between large slips, as anticipated by examining the upper panel of Figure 8 at $x=60$ and $180 \mathrm{~km}$. The bottom panels of Figures 9 and 10, for the more refined grid, show that this complexity has largely disappeared in favor of essentially periodic earthquakes with similar characteristics at both locations along strike. (The refined grid solution was done by beginning with a solution for the first 500 years on the less refined grid. Each cell of the less refined grid was then divided into four and given the same stress and velocity as the parent cell (these are the variables of the governing equations). This leads to a rapid readjustment of the velocity field within each cell group which makes $\delta$, defined as the time integral of $V$, develop some kinks in its profiles, as seen. These persist as an artifact of the grid change but have no effect on the subsequent velocity profiles, which are smooth and without kinks.)

Figures 11-13 show further 3-D results from three different grids for a case with $L=40 \mathrm{~mm}$ and $p=\max$ (hydrostatic, $\sigma_{n}-1000$ bars [100 MPa]), so that $h^{*}=2.0 \mathrm{~km}$. These are based on radiation damping $\eta=10^{2} \mu / 2 c$, lower by $10^{4}$ than the previous case, and take a large amount of computer time; thus results are shown only over limited times for the more refined grids. Figure 11 is for $N_{\text {length }}=64$ and $N_{\text {depth }}=8$, so that $h / h^{*}=1.5$; Figure 12 is for $N_{\text {length }}=128$ and $N_{\text {depth }}=$ 16 , so that $h / h^{*}=0.75$; Figure 13 is for $N_{\text {length }}=256$ and $N_{\text {depth }}=32$, so that $h / h^{*}=0.38$. The top panel of each figure shows the depth-averaged slip $\bar{\delta}(x, t)$ versus distance $x$ along strike at 5 -year intervals, and the bottom panel shows the logarithm of the maximum velocity in any cell within the model as a function of time, as sampled at time intervals of 0.01 year. (Because of time requirements, the intermediate grid was run for only a little more than $\mathbf{3 0 0}$ years, and the most refined grid was done by using the results from the intermediate grid at 300 years, dividing cells into four as discussed above, and continuing the calculation on the most refined grid for another 200 years.

Comparing successively (in Figures 11, 12, and 13) both the profiles of thickness-averaged slip and the maximum slip velocity records, it is seen clearly that spatio-temporal complexity disappears with grid refinement. Further, its disappearance is again comprehensible in terms of the different cell sizes $h$ involved when compared to $h^{*}$.

In order to document the transition from complexity to limit cycle behavior with feasible problem sizes, the previous 3-D examples involved only slightly oversized cells, with $h / h^{*}=1.9$ or 1.5 . Figure 14 shows the complex slip which results for a case with more strongly oversized cells, having $h / h^{*}=6.0$. This case is for the 2048 cell grid $\left(N_{\text {length }}=128\right.$ and $N_{\text {depth }}=16$ ) and is based on parameters $L=2.5 \mathrm{~mm}$ and $p=\max$ (hydrostatic, $\sigma_{n}-500$ bars $[50 \mathrm{MPa}]$ ), so that $h^{*}=0.25 \mathrm{~km}$, and $\eta=10^{6} \mu / 2 c$. The depth-averaged slip $\bar{\delta}(x, t)$ is shown as a function of distance $x$ along strike in the top panel, and slip $\delta(x, z, t)$ at $x=0.25 X_{\text {length }}=60 \mathrm{~km}$ is shown as a function of depth $z$ in the bottom panel. The 

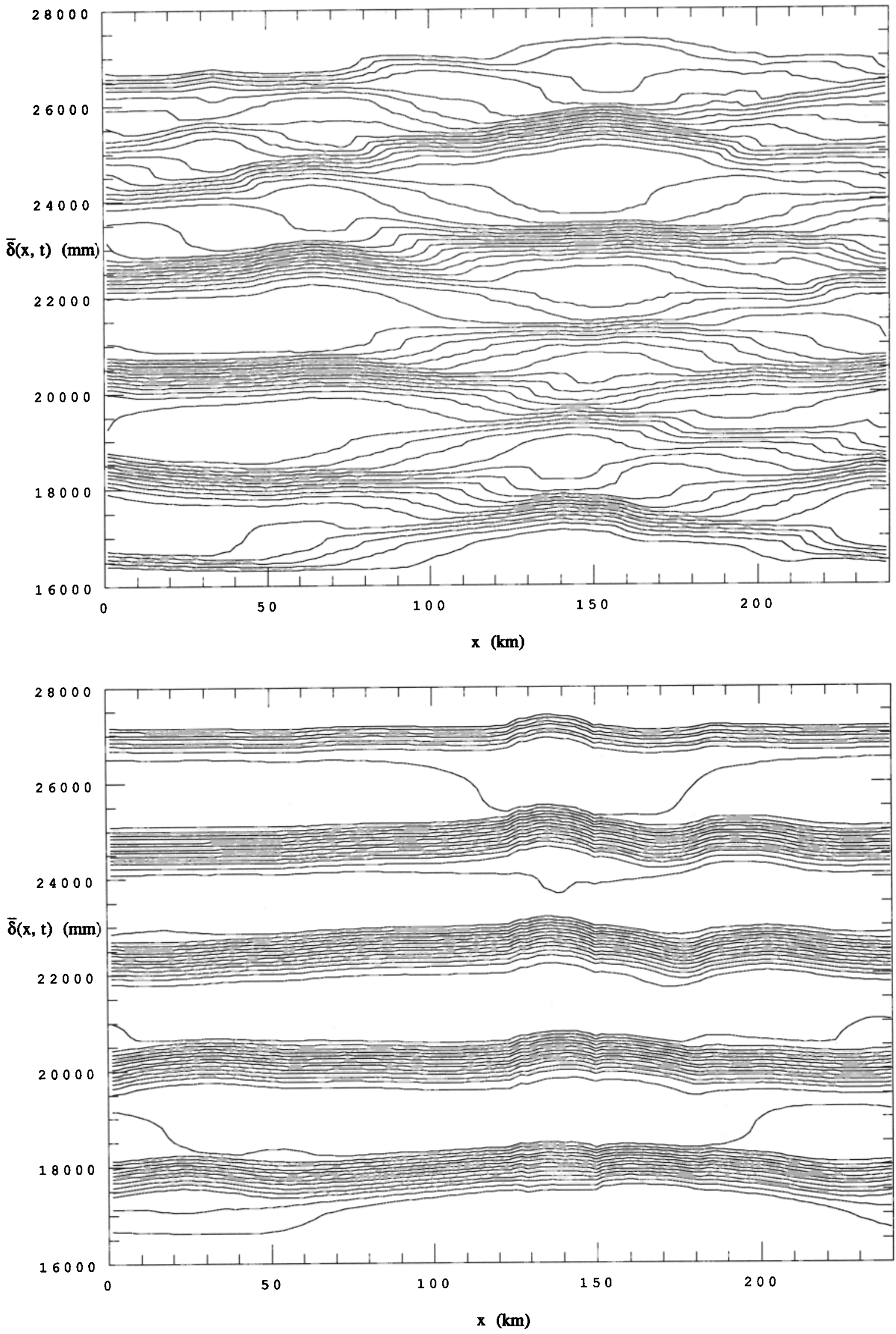

Fig. 8. Slip $\delta(x, z, t)$ averaged in $z$ over $24 \mathrm{~km}$ depth (i.e., $\delta(x, t))$ shown in millimeters on vertical axis versus distance $x$ in kilometers on horizontal axis over one $240-\mathrm{km}$ period along strike, drawn every 5 years. For model of Figure 1 with $L=40 \mathrm{~mm}, p=$ hydrostatic, $\eta=10^{6} \mu / 2 c$. (Top) For 2048 cells; 16 cells over $24 \mathrm{~km}$ depth, 128 columns of cells $\left(h / h^{*}=1.9\right)$. (Bottom) For 8192 cells; 32 cells over $24 \mathrm{~km}$ depth, 256 columns of cells $\left(h / h^{*}=0.95\right)$. 

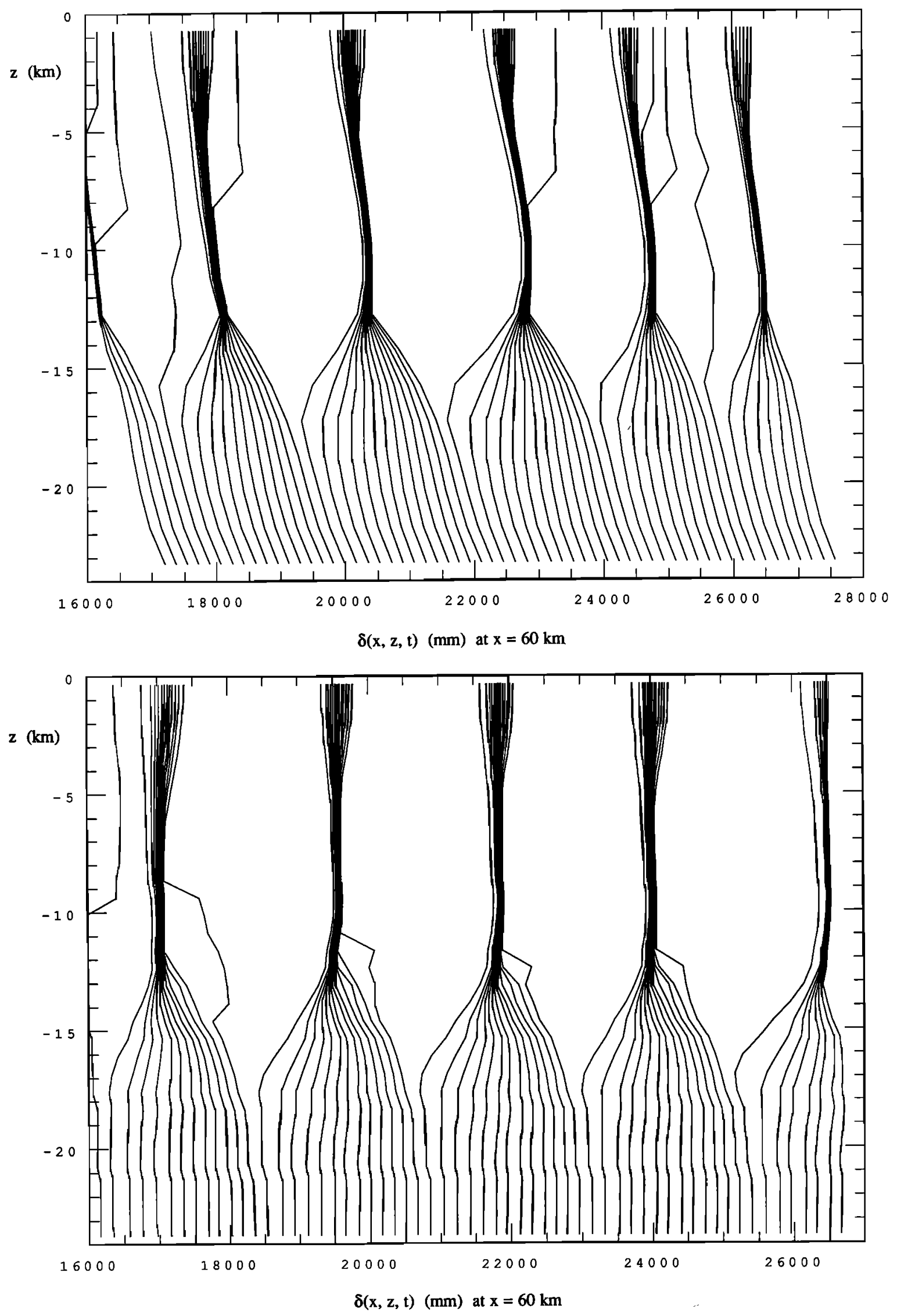

Fig. 9. Slip $\delta(x, z, t)$, at $x=60 \mathrm{~km}$, in millimeters on the horizontal axis versus depth $z$ in kilometers on the vertical axis, drawn every 5 years; for $L=40 \mathrm{~mm}, p=$ hydrostatic, $\eta=10^{6} \mu / 2 c$. (Top) For 2048 cells; 16 cells over $24 \mathrm{~km}$ depth, 128 columns of cells $\left(h / h^{*}=1.9\right)$. (Bottom) For 8192 cells; 32 cells over $24 \mathrm{~km}$ depth, 256 columns of cells $\left(h / h^{*}=0.95\right)$. 


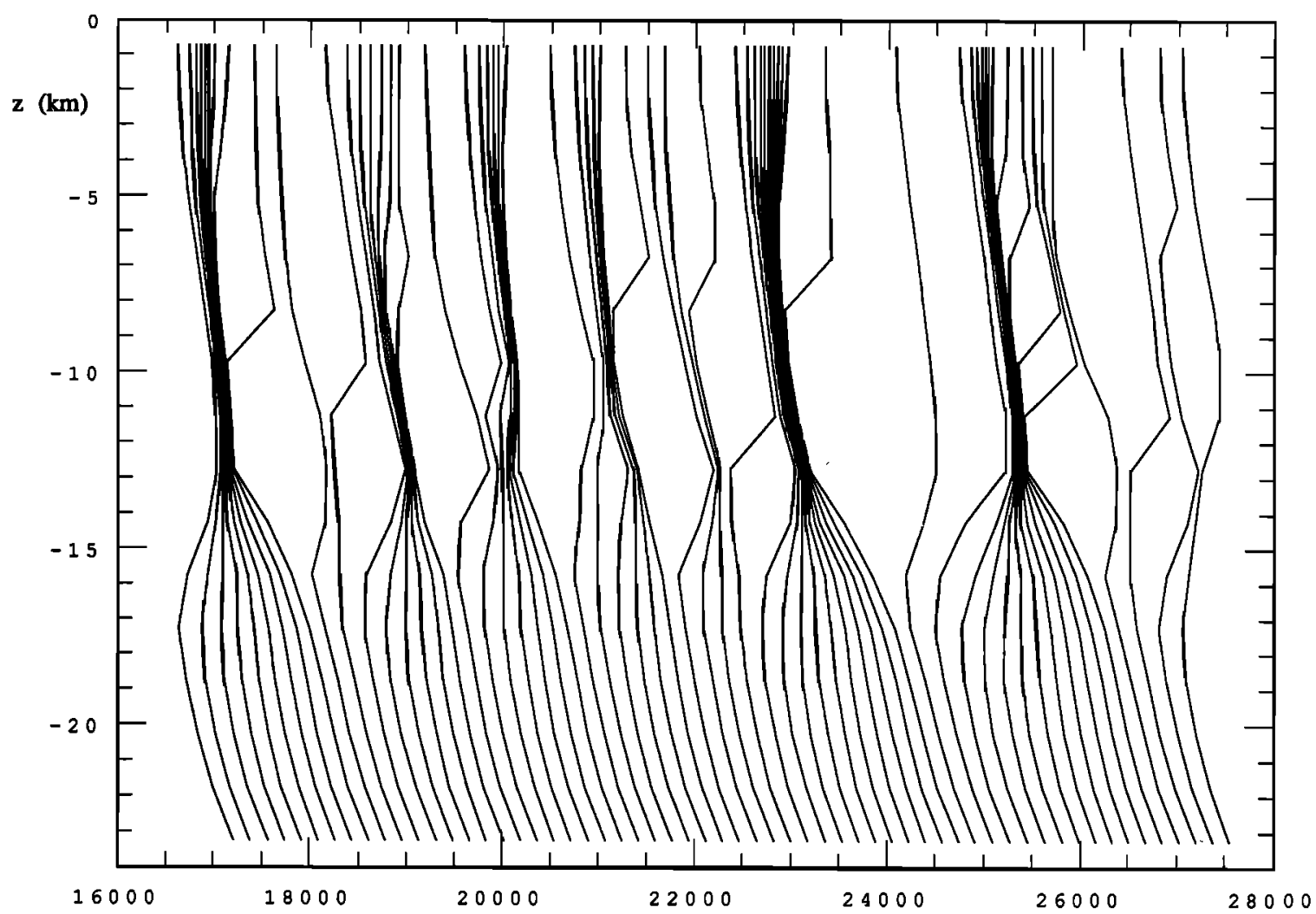

$\delta(\mathrm{x}, \mathrm{z}, \mathrm{t})(\mathrm{mm})$ at $\mathrm{x}=180 \mathrm{~km}$

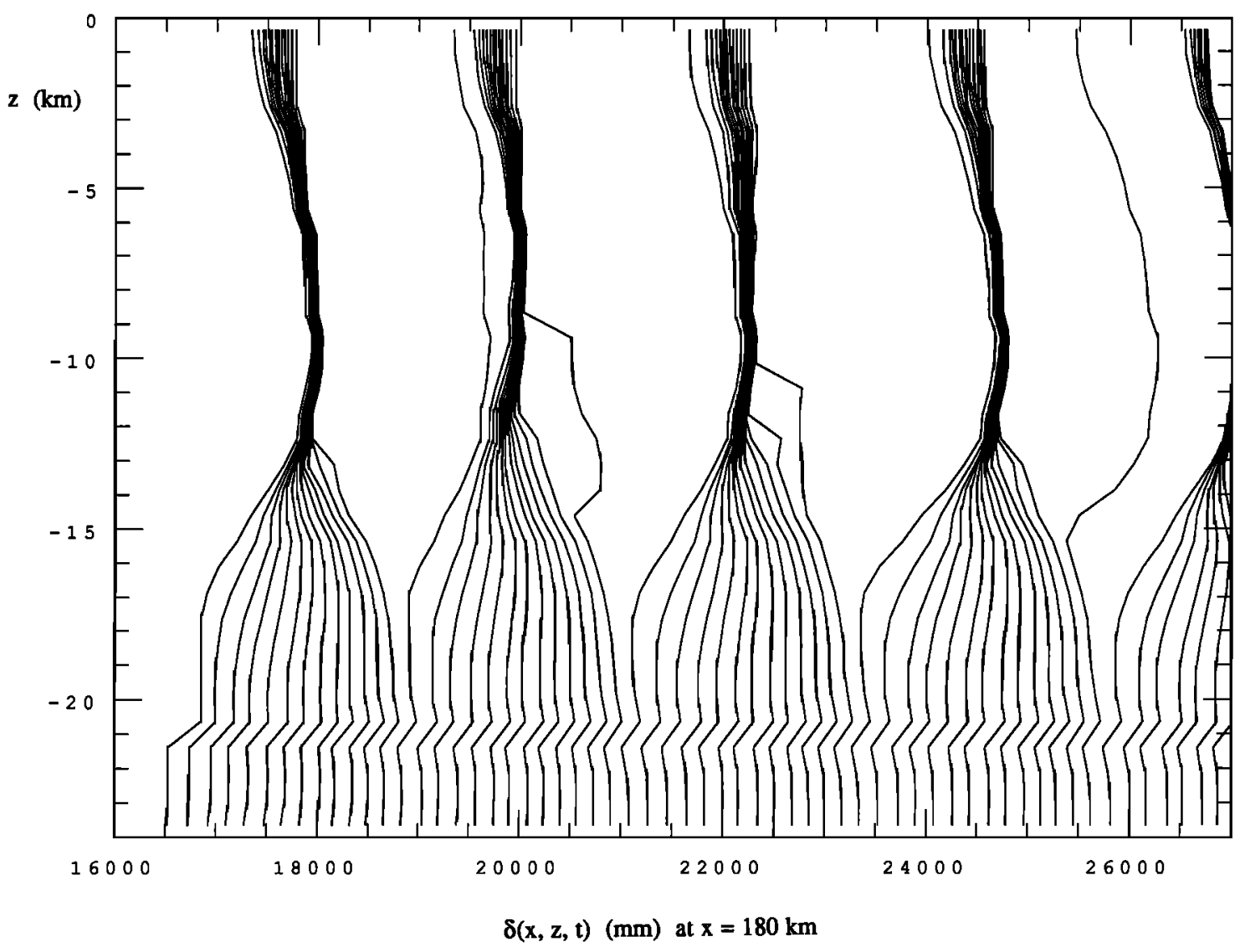

Fig. 10. Slip $\delta(x, z, t)$, at $x=180 \mathrm{~km}$, in millimeters on the horizontal axis versus depth $z$ in kilometers on the vertical axis, drawn every 5 years; for $L=40 \mathrm{~mm}, p=$ hydrostatic, $\eta=10^{6} \mu / 2 c$. (Top) For 2048 cells; 16 cells over $24 \mathrm{~km}$ depth, 128 columns of cells $\left(h / h^{*}=1.9\right)$. (Bottom) For 8192 cells; 32 cells over $24 \mathrm{~km}$ depth, 256 columns of cells $\left(h / h^{*}=0.95\right)$. 

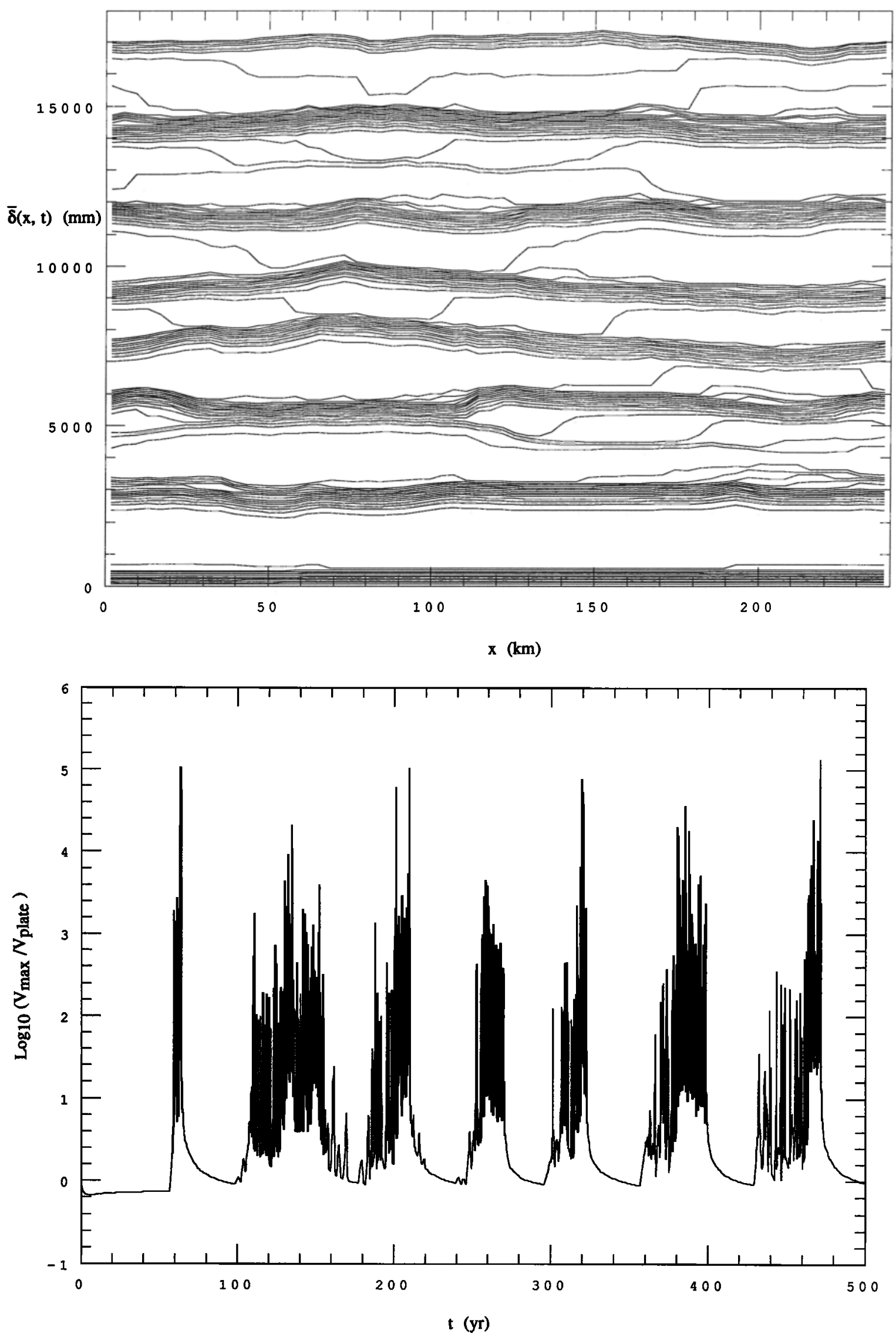

Fig. 11. Results for 512 cell grid $\left(N_{\text {length }}=64, N_{\text {depth }}=8\right.$ ), $h / h^{*}=1.5 ; L=40 \mathrm{~mm}, p=\max$ (hydrostatic, $\sigma_{n}$ -1000 bars [100 MPa]), $\eta=10^{2} \mu / 2 c$. (Top) Depth-averaged slip $\bar{\delta}(x, t)$ in millimeters on vertical axis versus distance $x$ along strike in kilometers on horizontal axis, drawn every 5 years. (Bottom) Maximum value over all cells of $\log _{10}$ $\left\{[\partial \delta(x, z, t) / \partial t] / V_{\text {plate }}\right\}$, as sampled every 0.01 year, on vertical axis versus time in years on horizontal axis. 

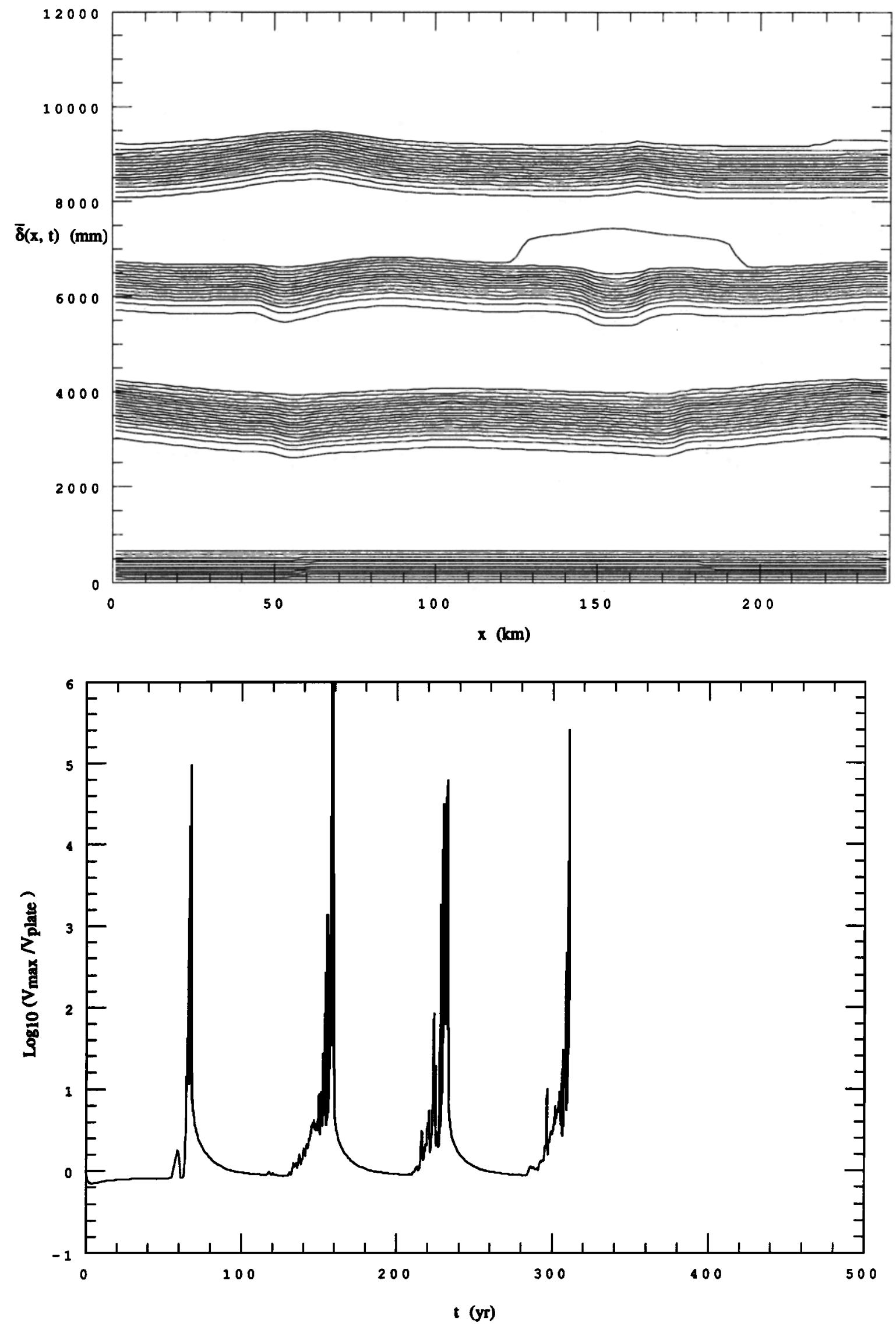

Fig. 12. Results for 2048 cell grid $\left(N_{\text {length }}=128, N_{\text {depth }}=16\right), h / h^{*}=0.75 ; L=40 \mathrm{~mm}, p=\max$ (hydrostatic, $\sigma_{n}-1000$ bars [100 MPa]), $\eta=10^{2} \mu / 2 c$. (Top) Depth-averaged slip $\bar{\delta}(x, t)$ in millimeters on vertical axis versus distance $x$ along strike in kilometers on horizontal axis, drawn every 5 years. (Bottom) Maximum value over all cells of $\log _{10}\left\{[\partial \delta(x, z, t) / \partial t] / V_{\text {plate }}\right\}$, as sampled every 0.01 year, on vertical axis versus time in years on horizontal axis. 

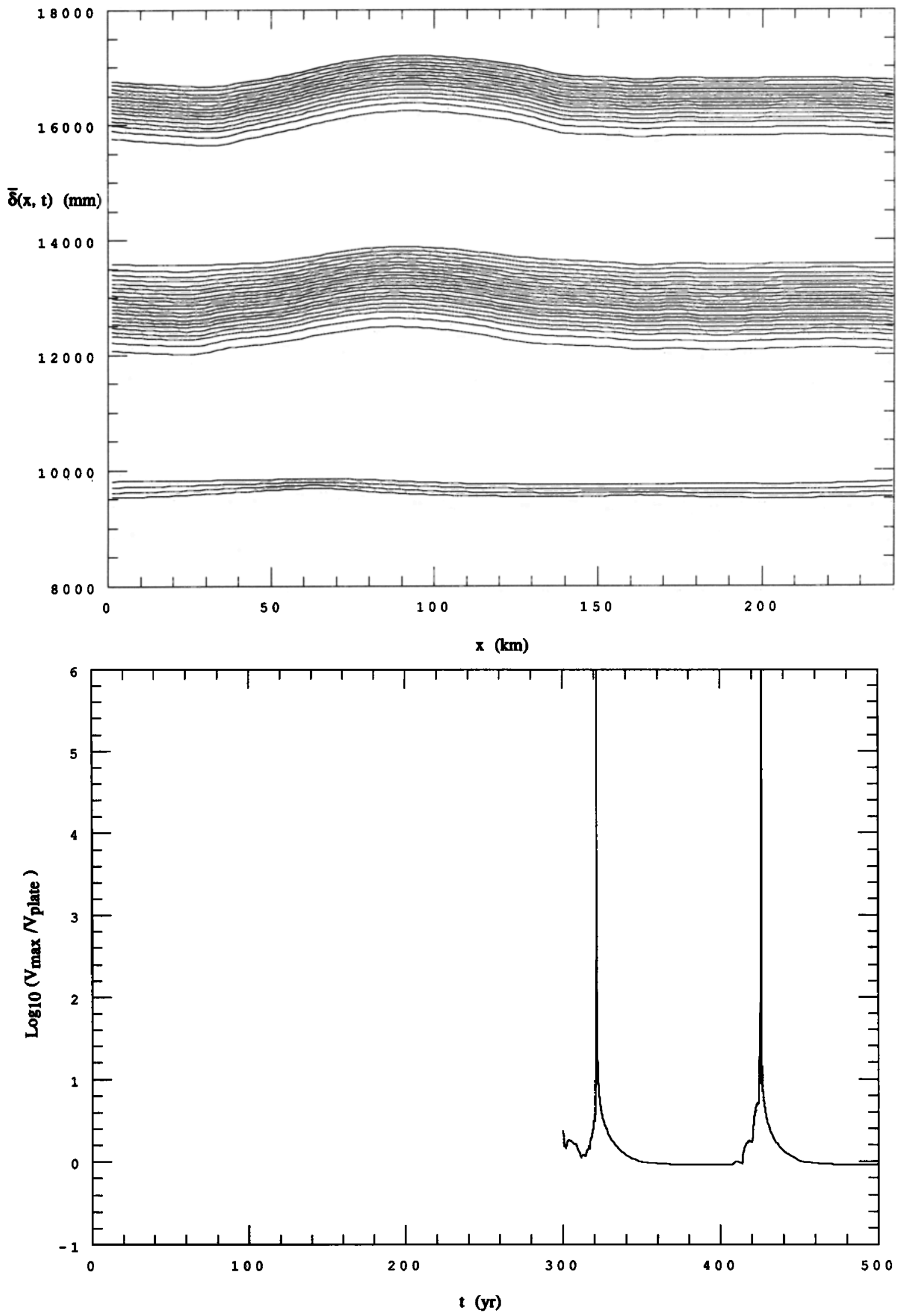

Fig. 13. Results for 8192 cell grid $\left(N_{\text {length }}=256, N_{\text {depth }}=32\right), h / h^{*}=0.38 ; L=40 \mathrm{~mm}, p=\max$ (hydrostatic, $\sigma_{n}-1000$ bars [100 MPa]), $\eta=10^{2} \mu / 2 c$. (Top) Depth-averaged slip $\bar{\delta}(x, t)$ in millimeters on vertical axis versus distance $x$ along strike in kilometers on horizontal axis, drawn every 5 years. (Bottom) Maximum value over all cells of $\log _{10}\left\{[\partial \delta(x, z, t) / \partial t] / V_{\text {plate }}\right\}$, as sampled every 0.01 year, on vertical axis versus time in years on horizontal axis. 

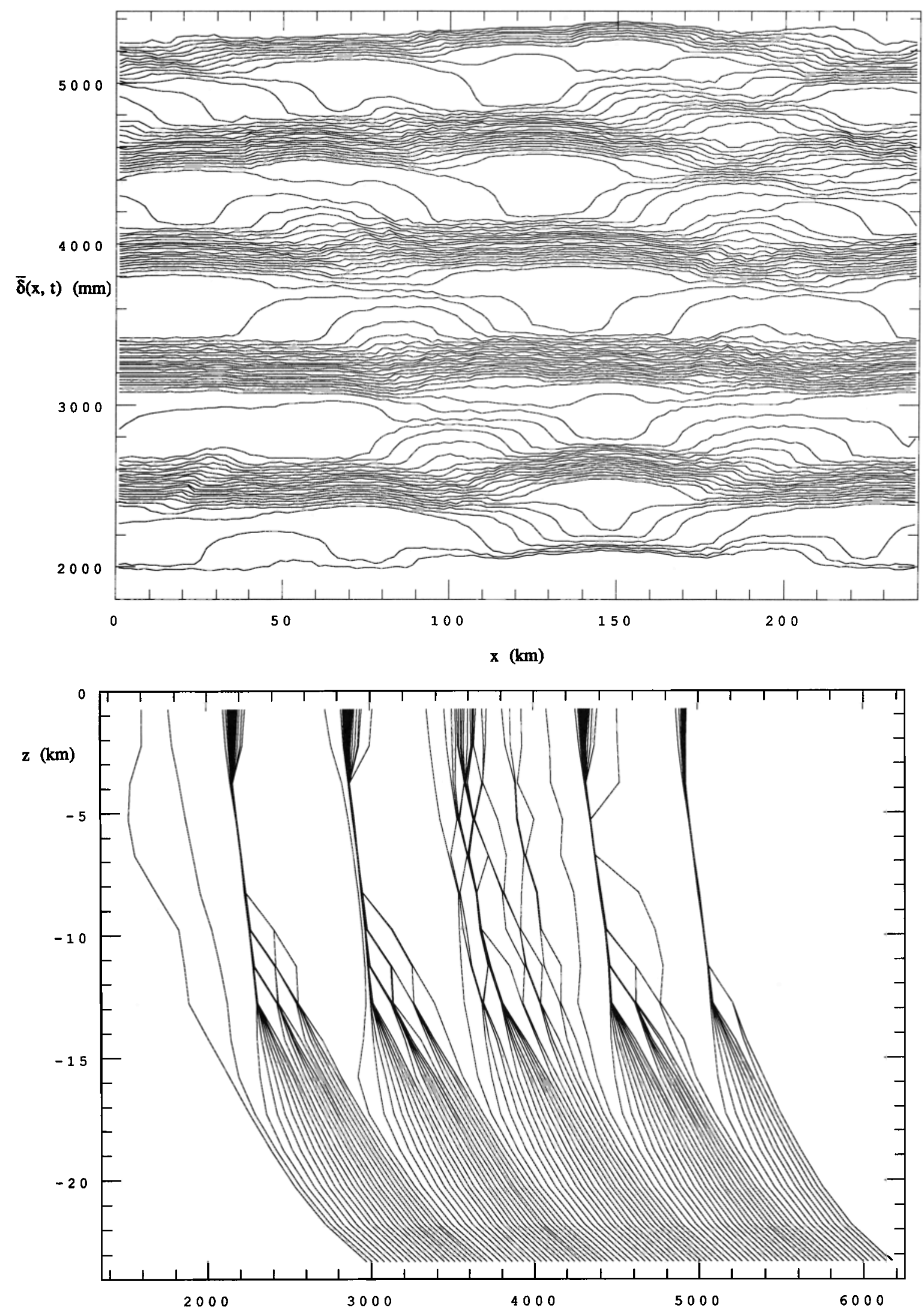

$\delta(\mathrm{x}, \mathrm{z}, \mathrm{t})(\mathrm{mm})$ at $\mathrm{x}=60 \mathrm{~km}$

Fig. 14. Complex slip based on a grid of strongly oversized cells, $h / h^{*}=6.0 ; 2048$ cell grid $\left(N_{\text {length }}=128, N_{\text {depth }}\right.$ $=16$ ) $L=2.5 \mathrm{~mm}, p=\max$ (hydrostatic, $\sigma_{n}-500$ bars $[50 \mathrm{MPa}]$ ), $\eta=10^{6} \mu / 2 c$. Slip drawn every 1 year from results over the time period $t=100$ years to $t=191$ years. (Top) Depth-averaged slip $\bar{\delta}(x, t)$ in millimeters on vertical axis versus distance $x$ along strike in kilometers on horizontal axis. (Bottom) Slip $\delta(x, z, t)$, at $x=60 \mathrm{~km}$, in millimeters on the horizontal axis versus depth $z$ in kilometers on the vertical axis. 
functions are printed out every year for the 91-year period from $t=100$ years to $t=191$ years. Such complexity seems to be typical of results with strongly oversized cells. Note that despite the complexity, the rupture process is still coordinated along strike, with all portions of the fault zone undergoing large slip over time intervals of the order of 5 years (about $25 \%$ of the average recurrence time in this case). Thus an increase in slip rate through cell instabilities in one part of the fault zone is, in this case, a good but imperfect predictor that cells in other parts of the fault zone will soon be active. The style of the instabilities at any particular section along strike involves an alteration between motion of the brittle part of the fault zone in single large events and in sequences of smaller events, as shown for the section at $x=60 \mathrm{~km}$.

Because of the small $L$ value, the calculation for Figure 14 ran slowly. It was originally tried with $\eta=10^{4} \mu / 2 c$ and required approximately 14 days of Sparcstation $2 \mathrm{cpu}$ time to run through 105 years, taking $7.2 \times 10^{5}$ time increments. The version with $\eta=10^{6} \mu / 2 c$ gave qualitatively similar results but covered about 2.5 times as many years per unit of cpu time; 5 days cpu and $2.6 \times 10^{5}$ time increments were required to cover the 91 years of history shown. It is likely that improvements in programming could be implemented which would significantly reduce these cpu times.

\section{Discussion and Conclusions}

A model for determining slip histories along a planar vertical fault embedded in an elastic half-space (Figure 1) has been analyzed. Fault zone constitutive relations are of the laboratory-based rate- and state-dependent friction type and have the feature of a characteristic slip distance $L$ for slip weakening or frictional state evolution (Figure 2). They are implemented in the context of depth-variable temperature, normal stress, and pore pressure (Figures 3 and 4). The analysis is based on quasi-static elasticity, rather than on a proper elastodynamic formulation, and what would otherwise be unbounded slip rates during instabilities are limited to values scaling with stress drop by the inclusion of a viscous term that approximately represents energy outflow as seismic radiation during rapid unstable slip.

The problem described by the model is posed numerically on a grid of cells and a criterion is derived for the cell size $h$ to be small enough for the discrete numerical model to give a valid solution of the underlying continuous equations. This is that $h$ be small compared to $h^{*}$, where $h^{*}=2 \mu L / \pi(B-$ $A)_{m}, \mu$ is the elastic shear modulus, $(B-A)_{m}$ is the maximum value of $\left[-V d \tau^{\mathrm{ss}}(V) / d V\right]$ over the fault plane, $V$ is local slip rate, and $\tau^{s s}(V)$ is the steady state fictional strength at that rate (Figure 2). In the various numerical examples shown here, $h^{*}$ ranges from $2 \times 10^{4} L$ [hydrostatic pore pressure; $(B-A)_{m}=10$ bars $\left.(1.0 \mathrm{MPa})\right]$ to $10^{5} L$ (elevated pore pressure, offset by 500 bars $(50 \mathrm{MPa})$ from normal stress; $(B-A)_{m}=2$ bar $\left.(0.2 \mathrm{MPa})\right)$. Cells of size larger than $h^{*}$ can fail independently of one another, whereas those with $h \ll h^{*}$ are too elastically stiff to fail unstably alone and can do so only as part of a cooperatively slipping array of cells.

Computational limits based on present algorithms and machines prohibit solutions to the model with suitably refined grids unless $L$ is larger than about $10 \mathrm{~mm}$ in 3-D simulations and about $2 \mathrm{~mm}$ in 2-D simulations. Neverthe- less, in every case for which it has been computationally feasible to explore the issue, the following is found: While the results of the computations with oversized cells $\left(h>h^{*}\right)$ show richly complex slip, with a spectrum of event sizes, that complexity disappears in favor of simple limit cycles of repeated large earthquakes as the cell size is diminished, relative to $h^{*}$, so as to properly solve the underlying continuous equations. This is documented by the comparisons made in Figures 5-14, where the effect of reduction in cell size is to cause a transition from spatio-temporally complex slip to simple limit cycles; the response can always be understood in terms of the ratio $h / h^{*}$, as given with each figure. Large $h / h^{*}$ compared to unity leads to disordered slip, small $h / h^{*}$ leads to ordered slip.

These results are at conflict with the emergent view that spatio-temporally complex slip behavior, with features sometimes described as self-organized criticality, is a generic outcome of mechanical fault models. Just the opposite occurs here; the generic result of actual solutions of the governing equations, rather than of an oversized cell approximation to them, is simple limit cycles of repeated large earthquakes. The stress concentration at the front of a spreading zone of slip, when not masked by the use of oversized cells, seems to be too compelling for an earthquake rupture to stop until it has spread entirely over the fault plane.

The results here raise concerns about conclusions on spatio-temporal complexity drawn on the basis of fault models with ad hoc discretizations, whether into an array of spring-connected rigid blocks or as a cellular automata. Such models as discussed earlier may be said to be inherently discrete, in that they do not have a well-defined continuum limit. That is because the models have adopted friction laws equivalent to setting $L=0$, i.e., to pure velocity weakening or its simplified form as distinct static and kinetic friction but without attributes such as slip weakening, displacementdependent state transition, or fracture energy. Thus the critical cell size $h^{*}=0$ for those models, and by having discarded the short-wavelength constitutive behavior, they fail to predict the transition of slip response documented here when discrete element size is reduced below $h^{*}$. Spatio-temporally complex slip, with self-similar distributions of small (compared to overall system size) events, does seem to be a generic feature of those inherently discrete models which do not have a continuum limit.

An unresolved issue in the present study is that of whether a more precise treatment of elastodynamics during instabilities would qualitatively change the results. Such must be considered because the wave-mediated arrest of a dynamic slip event may leave a heterogeneous distribution of residual static stress on the ruptured surface. It is possible that such heterogeneity, if strong enough, could affect the nucleation and arrest locations of subsequent ruptures and could be a mechanism for sustaining permanently complex seismic behavior, showing a spectrum of event sizes. Recent numerical simulations of spontaneous faulting in an elastic continuum by Madariaga and Cochard [1992] have addressed the problem of stress heterogeneity resulting from wavemediated rupture arrest. They assume pure velocity weakening, equivalent to $L=0$. Their results for friction models with no, or little, continuing velocity weakening at seismic slip rates show generally smooth final stress on the rupture that is locally depressed where arrest waves, propagating 
back from blocked tips of the rupture, meet one another. However, their results based on velocity weakening that remains significant at speeds near seismic rates show markedly heterogeneous, rapidly fluctuating final stress; the scale of its fluctuation seems comparable to the numerical grid spacing, and the results must therefore be regarded as tentative until it is understood if and how they are modified by use of a regularized velocity weakening law, with a nonzero slip weakening or state transition length dimension $L$.

Stress heterogeneity resulting from the dynamics of rupture propagation and arrest seems to be important to the sustained complexity of response of Burridge-Knopoff models, as recognized by Carlson and Langer [1989] and Carlson et al. [1991], although it is difficult to know how important results from the B-K model are for reasons noted earlier. The dynamic interrelation between the stress and slip histories are fundamentally different for systems with concentrated mass along the fault than for faults embedded in a continuum, dynamic overshoot effects are greatly overemphasized by the lack of energy radiation into an adjoining continuum, and the simple stress interactions of the B-K model do not concentrate stress ahead of a rupture in a manner that scales the stress concentration with rupture size, like for cracks embedded in a surrounding elastic continuum, so that larger ruptures do not properly become increasingly difficult to stop. It also remains unknown as to how much the dynamics of the B-K class of models would be influenced by use of friction laws with a nonzero $L$, with the block spacing sufficiently reduced in size relative to a distance analogous to $h^{*}$ for the B-K model, so as to simulate a continuum limit. The inclusion of even an arbitrarily small but nonzero fracture energy, as would be associated with nonzero $L$, in the continuum version of the B-K model is known to rule out supersonic rupture propagation that is sometimes otherwise allowed by the model.

It seems that the models with arrays of identical springconnected blocks or cells were proposed with the intention of modeling a uniform fault and that it was tacitly assumed that no essential feature of the physics was lost either in the discretization of an a priori conceptual model into cells or spring-connected blocks or in the neglect of slip-weakening response features involving a finite $L$. The present results raise the possibility that if those models were reformulated with a nonzero $L$ and if the cell size or block spacing were continuously reduced in size, with element-to-element stress interactions chosen to be faithful to the response of a fault in an elastic continuum (and if continuum rather than concentrated mass Newtonian dynamics were simulated in the dynamical models), then qualitatively different results might emerge, lacking the spatio-temporal complexity that has been reported.

Thus a tentative conclusion suggested, but not proven, by the present results is that spatio-temporally complex slip. resembling earthquake processes, will not emerge from physically justifiable modeling of the nonlinear dynamics of frictional slip on a geometrically smooth fault surface. It is suggested that consideration of the geometric disorder of fault zones, including distinct fault segments which join at bends or offsets and can arrest ruptures [Bakun, 1980; Lindh and Boore, 1981; Schwartz and Coppersmith, 1984; King and Nabelek, 1985; King, 1986; Sibson, 1986], and perhaps also the fractal-like surface roughness of faults [Power et al.,
1988; Power and Tullis, 1991], will be necessary to provide a physically viable description of seismic complexity.

Results shown here that are based on oversized cells, relative to $h^{*}$, seem to be a better mirror of observed slip complexity than are those corresponding to a grid that is sufficiently refined to be approaching the continuum limit, that is, to a grid which allows one to actually solve the mathematical problem posed. The critical feature may be that such oversized cells can fail independently of one another. In this respect, adjacent oversized cells may provide a crude model of adjacent fault segments which can fail independently of one another due to difficulties of transmitting unstable slip rupture across geometric complexities where such segments join. The proper understanding of spatio-temporally complex slip may then lie in clarifying the interaction of slip-weakening sensitive features of fault zone response, having a characteristic scale length proportional to $L \mu /(B-A)$ (i.e., to $\left.h^{*}\right)$, with geometric disorder. Based on the various results for oversized cells presented here, it seems likely that geometric disorder of a fault zone at scales larger than $h^{*}$ will cause its dynamics at those scales to mimic the dynamics of an inherently discrete system and hence to show the spatio-temporally complex slip that seems to be generic to such systems.

An open question is that of whether continued geometric disorder at yet larger scales is necessary for complex slip at those larger scales. That seems not to be necessary in the simple cellular automata models, including quasi-static implementations of the B-K model on a 2-D fault surface, in that one cell size suffices to give complex failure over all sizes. Such might occur only because the simple models are unphysical in failing to scale the stress concentration at the edge of a slipping zone with the size of that zone, as required by elasticity theory.

Additional evidence that slip complexity and geometric disorder are related comes from Wesnousky $[1988,1990]$. He shows that seismic productivity of fault zones (in generating many small earthquakes) and geometric disorder as measured by stepovers along fault traces are strongly correlated with one another. By studying five distinct faults in southern California, including the San Andreas and NewportInglewood faults, as well as the Calaveras fault in central California and the North Anatolian fault in Turkey, he shows that geometric disorder of a fault zone diminishes with cumulative slip over its geological history. The NewportInglewood and San Andreas faults are at the extremes of his data set and differ by a factor of about 50 in disorder as measured by either the number of stepovers of the fault trace in excess of $1 \mathrm{~km}$ per unit length along strike or the cumulative sum of absolute stepover distances per unit length along strike. In a study involving only the southern California faults, Wesnousky [1990] also shows that the seismic productivity of a fault zone is a similar diminishing function of cumulative geological slip. The productivity is defined as the number of events with magnitude above 3.0 per unit length of fault, divided by the long-term slip rate of the fault for normalization. For this quantity the NewportInglewood and San Andreas faults are again at the extremes of his data set; with its high geometric disorder as measured by stepovers, the less slipped Newport-Inglewood fault is about 100 times more productive seismically than is the highly slipped and geometrically less disordered San Andreas fault. 


\section{Appendix: Notes on Numerical Formulation}

The stiffness component $k_{i j}$ is defined as the negative of the stress $\tau$ induced at the center of cell $i$ by unit slip $\delta$ in the source cell $j$ and in the periodic repeats of that cell along strike, spaced by $X_{\text {length }}$. Chinnery [1963] gave the solution for the displacement field for uniform slip on a rectangular dislocation surface in an elastic half-space. The relevant stress component may be extracted from that solution by the expression $\tau=\mu\left(\partial u_{x} / \partial y+\partial u_{y} / \partial x\right)$, and Chinnery has derived the result in equations communicated personally by him to Stuart [1986] in 1983 for the Poisson solid, $\nu=0.25$. Stuart and T. E. Tullis, who have used the equations in a similar 3-D program with rate- and state-dependent friction have kindly provided the equations (W. D. Stuart and T. E. Tullis, personal communication, 1990), and, in the present notation, they are as follows: Coordinates of the vertices of the source cell, which has side lengths $h / q$ in the $x$ direction and $h$ in the $z$ direction and has its center at $x=x_{\text {cell } j}$ and $z=z_{\text {cell } j}$, are

$$
\begin{array}{cc}
x_{1}=x_{\text {cell } j}+h / 2 q, & x_{2}=x_{\text {cell } j}+h / 2 q, \\
x_{3}=x_{\text {cell } j}-h / 2 q, & x_{4}=x_{\text {cell } j}-h / 2 q \\
z_{1}=z_{\text {cell } j}+h / 2, & z_{2}=z_{\text {cell } j}-h / 2, \\
z_{3}=z_{\text {cell } j}+h / 2, & z_{4}=z_{\text {cell } j}-h / 2 .
\end{array}
$$

Then, introducing the parameters

$$
t_{\alpha}=x_{\alpha}-x_{\text {cell } i}, \quad q_{\alpha}=z_{\alpha}-z_{\text {cell } i}, \quad p_{\alpha}=z_{\alpha}+z_{\text {cell } i}
$$$$
(\alpha=1,2,3,4) \text {, }
$$

where the center of cell $i$ is at $x=x_{\text {cell } i}$ and $z=z_{\text {cell } i}$, the following three functions are defined:

$$
\begin{aligned}
F_{\alpha}= & 1 /\left\{\left[\left(t_{\alpha}\right)^{2}+\left(q_{\alpha}\right)^{2}\right]^{1 / 2}\left[\left(\left(t_{\alpha}\right)^{2}+\left(q_{\alpha}\right)^{2}\right)^{1 / 2}+q_{\alpha}\right]\right\} \\
& +1 /\left\{\left[\left(t_{\alpha}\right)^{2}+\left(p_{\alpha}\right)^{2}\right]^{1 / 2}\left[\left(\left(t_{\alpha}\right)^{2}+\left(p_{\alpha}\right)^{2}\right)^{1 / 2}+p_{\alpha}\right]\right\} \\
G_{\alpha}= & \left\{(1 / 4)\left[\left(t_{\alpha}\right)^{2}+\left(p_{\alpha}\right)^{2}\right]^{1 / 2}+q_{\alpha}\right\} /\left\{\left[\left(t_{\alpha}\right)^{2}+\left(p_{\alpha}\right)^{2}\right]^{1 / 2}\right. \\
& \left.\cdot\left[\left(\left(t_{\alpha}\right)^{2}+\left(p_{\alpha}\right)^{2}\right)^{1 / 2}+p_{\alpha}\right]^{2}\right\}-\left[\left(p_{\alpha}\right)^{2}-\left(q_{\alpha}\right)^{2}\right]\left\{2 \left[\left(t_{\alpha}\right)^{2}\right.\right. \\
+ & \left.\left.\left(p_{\alpha}\right)^{2}\right]^{1 / 2}+p_{\alpha}\right\} /\left\{2 \left[\left(t_{\alpha}\right)^{2}\right.\right. \\
+ & \left.\left.\left(p_{\alpha}\right)^{2}\right]^{3 / 2}\left[\left(\left(t_{\alpha}\right)^{2}+\left(p_{\alpha}\right)^{2}\right)^{1 / 2}+p_{\alpha}\right]^{2}\right\}, \\
H_{\alpha}= & q_{\alpha} /\left\{\left[\left(t_{\alpha}\right)^{2}+\left(q_{\alpha}\right)^{2}\right]^{1 / 2}\left[\left(\left(t_{\alpha}\right)^{2}+\left(q_{\alpha}\right)^{2}\right)^{1 / 2}+t_{\alpha}\right]\right\} \\
& +p_{\alpha} /\left\{\left[\left(t_{\alpha}\right)^{2}+\left(p_{\alpha}\right)^{2}\right]^{1 / 2}\left[\left(\left(t_{\alpha}\right)^{2}+\left(p_{\alpha}\right)^{2}\right)^{1 / 2}+t_{\alpha}\right]\right\} .
\end{aligned}
$$

The stiffness associated with a single source cell $i$ (i.e., not yet considering the infinite number of periodic repeats of the source cell along strike) is then

$$
\begin{aligned}
& k\left(x_{\text {cell } i}, z_{\text {cell } i} ; x_{\text {cell } j}, z_{\text {cell } j}\right) \\
&=(\mu / 2 \pi) \sum_{\alpha=1}^{4}(-1)^{\alpha}\left\{(2 / 3) t_{\alpha}\left(F_{\alpha}+G_{\alpha}\right)+(1 / 2) H_{\alpha}\right\}
\end{aligned}
$$

and the stiffness for the periodically repeated source is

$$
k_{i j}=\sum_{m=-\infty}^{+\infty} k\left(x_{\text {cell } i}, z_{\text {cell } i} ; x_{\text {cell } j}+m X_{\text {length }}, z_{\text {cell } j}\right)
$$

In programming this result, the sum on $m$ is extended from -10 to 10 . If multiplied by a cell area, $[k]$ would have the same units of what is more commonly called a stiffness matrix, i.e., a matrix relating work conjugate sets of displacements and forces. Such a matrix relating work conjugates is necessarily symmetric for an elastic system. Because $[k]$ has been based on stress at a point (cell center), it is not precisely symmetric although each $k_{i j}$ and $k_{j i}$ are numerically close. Recognizing that a properly defined stiffness would be symmetric, $[k]$ is redefined as its symmetric part, which also simplifies the equation-solving routine.

The governing equations are extremely "stiff" and an implicit integration procedure was devised as follows. The basic idea is to integrate the constitutive law over each time step as if $V$ were constant during that step and to do so in a manner that would be correct for arbitrarily large time steps if $V$ in the present increment was unchanged from $V$ in the last, or changed by a sufficiently small amount that the result can be linearized in the change of $V$ from the present step to the last. Thus let $V_{i}^{t+\Delta t}$ denote the velocity of cell $i$ over the time step from $t$ to $t_{t}+\Delta t$, and let $\tau_{i}^{t+\Delta t}$ be the stress of that cell at the end of the time step; $V_{i}^{t}$ is the velocity in the previous time step and $\tau_{i}^{t}$ is the stress at the end of that previous time step. The slip in the time step from $t$ to $t+\Delta t$ is $\delta_{i}^{t+\Delta t}-\delta_{i}^{t}=V_{i}^{t+\Delta t} \Delta t$. The constitutive relation is then integrated for constant velocity over the step and put in the form

$$
\tau_{i}^{t+\Delta t}-\tau_{i}^{t}=-C_{i}^{t}+D_{i}^{t}\left[\left(V_{i}^{t+\Delta t}-V_{i}^{t}\right) / V_{i}^{t}\right]
$$

where the $C_{i}^{t}$ and $D_{i}^{t}$ are chosen so that result is correct, up to the term of first order in $\left(V_{i}^{t+\Delta t}-V_{i}^{t}\right) / V_{i}^{t}$, for arbitrarily large $\Delta t$ and are

$$
\begin{gathered}
C_{i}^{t}=\left[\tau_{i}^{t}-\tau_{i}^{\mathrm{SS}}\left(V_{i}^{t}\right)\right]\left[1-\exp \left(-V_{i}^{t} \Delta t / L\right)\right] \\
D_{i}^{t}=A_{i}-\left[A_{i}-V_{i}^{t} d \tau_{i}^{\mathrm{SS}}\left(V_{i}^{t}\right) / d V_{i}^{t}\right]\left[1-\exp \left(-V_{i}^{t} \Delta t / L\right)\right] \\
-\left[\tau_{i}^{t}-\tau_{i}^{\mathrm{SS}}\left(V_{i}^{t}\right)\right]\left(V_{i}^{t} \Delta t / L\right) \exp \left(-V_{i}^{t} \Delta t / L\right) .
\end{gathered}
$$

The relations expressing equilibrium, with the radiation damping term, are then written at times $t$ and $t+\Delta t$ as

$$
\begin{gathered}
\tau_{i}^{t+\Delta t}=-\sum_{j} k_{i j}\left[\delta_{j}^{t+\Delta t}-V_{\text {plate }}(t+\Delta t)\right]-\eta V_{i}^{t+\Delta t}, \\
\tau_{i}^{t}=-\sum_{j} k_{i j}\left(\delta_{j}^{t}-V_{\text {plate }} t\right)-\eta V_{i}^{t} .
\end{gathered}
$$

Subtracting the second from the first and using the above representation of $\tau_{i}^{t+\Delta t}-\tau_{i}^{t}$, one finds

$$
\begin{aligned}
\left(\eta V_{i}^{t}+D_{i}^{t}\right) V_{i}^{t+\Delta t} / V_{i}^{t} & +\Delta t \sum_{j} k_{i j} V_{j}^{t+\Delta t} \\
& =\eta V_{i}^{t}+D_{i}^{t}+C_{i}^{t}+\Delta t \sum_{j} k_{i j} V_{\text {plate }},
\end{aligned}
$$

a set of simultaneous equations to be solved for the velocity in each time step. The job now is to choose the time step and to solve the equations. The maximum time step allowed is 
that which makes $V_{i}^{t} \Delta t / L=1$ for the cell which is fastest moving at the start of the increment. A shorter time step is chosen if, upon finding the first positive value, if any, of $\Delta t$ for which either $\eta V_{i}^{t}+D_{i}^{t}$ or $\eta V_{i}^{t}+D_{i}^{t}+C_{i}^{t}$ vanish for each cell, and then choosing $\Delta t$ as half the smallest of those values, the resulting $\Delta t$ is shorter than the shortest defined by $V_{i}^{t} \Delta t / L=1$. The idea is to remain in a range for which the approximate integration of the constitutive relation over $\Delta t$ will remain valid.

The system of simultaneous equations is then solved by implementing the conjugate gradient iterative procedure, following an algorithm described by Golub and VanLoan [1983, p. 373], after preconditioning the system to the form $[a]\{x\}=\{b\}$, where

$$
\begin{gathered}
x_{i}=\left(V_{i}^{t+\Delta t}-V_{i}^{t}\right) / y_{i}, \quad a_{i j}=1 \text { when } i=j, \\
a_{i j}=k_{i j} y_{i} y_{j} \text { when } i \neq j, \\
b_{i}=\left[C_{i}^{t}+\Delta t \sum_{j} k_{i j}\left(V_{\text {plate }}-V_{j}^{t}\right)\right] y_{i} /(\Delta t)^{1 / 2}, \\
y_{i}=\left(V_{i}^{t} \Delta t\right)^{1 / 2} /\left(\eta V_{i}^{t}+D_{i}^{t}+k_{i i} V_{i}^{t} \Delta t\right)^{1 / 2} .
\end{gathered}
$$

In the 3-D cases, the multiplication $[k]\{V\}$ that is required to form $\{b\}$ is done with the aid of the fast Fourier transform. Because an element of $[k]$ corresponding to a given pair of cells is invariant to the position of that cell pair along strike, the matrix multiplication can be rewritten as the sum of a number (proportional to $\left(N_{\text {depth }}\right)^{2}$ ) of convolution summations involving arrays of size $N_{\text {length }}$. The FFT speeds the convolutions, so that the number of operations scales as $\left(N_{\text {depth }}\right)^{2} \quad N_{\text {length }} \log _{2}\left(N_{\text {length }}\right)$ rather than as $\left(N_{\text {depth }}\right)^{2}$ $\left(N_{\text {length }}\right)^{2}$. Each iteration in the conjugate gradient procedure also involves a matrix multiplication of $[k]$ with an iteration vector and is likewise hastened by use of the FFT. The iteration stops, and the solution for $\{x\}$ is accepted, when the norm (square root of sum of squares of components) of $\{b\}-$ $[a]\{x\}$ diminishes below $10^{-6}$ times the norm of $\{b\}$. This usually requires a small number of iterations, starting from an initial guess $\{x\}=\{0\}$, often as few as three and only rarely as many as $\mathbf{1 0 .}$

Finally, if the ratio $V_{i}^{t+\Delta r} / V_{i}^{t}$ passes outside certain limits, taken as $1 / 2<V_{i}^{t+\Delta t} / V_{i}^{t}<2$ in the cases shown, the $\Delta t$ that had been chosen is replaced by half its value and the conjugate gradient solution process starts anew for that reduced $\Delta t$. This situation seldom happens, and, indeed, the procedure to estimate an allowable time step was devised to avoid it.

Acknowledgments. These studies were supported by the U.S. Geological Survey/National Earthquake Hazards Reduction Program (grant 14-08-0001-G1788), the Southern California Earthquake Center (PO 569928 from University of Southern California), and the NSF National Center for Supercomputer Applications (Urbana, Illinois) through a grant of time on Cray 2 and Y-MP machines. I am grateful to Terry E. Tullis for discussion and assistance on organizing the original 3-D computer program, Donald G. M. Anderson for discussions on numerical methods, Mark F. Linker for discussions and help in running the program on various machines, and Charles G. Sammis for a question after a presentation [Rice, 1991] which sharpened my appreciation of the importance of cells failing independently of one another as a basis for differences between inherently discrete systems and those with a continuum limit; to Per Bak, Yehuda Ben-Zion, Jean M. Carlson, James H. Dieterich, Renata Dmowska, Leon Knopoff, James S. Langer, Bruce E. Shaw, and
Chao Tang for discussions on the origin of slip complexity in fault models; and to Raul Madariaga and another unidentified reviewer for comments which helped to improve the presentation.

\section{REFERENCES}

Bak, P., and C. Tang, Earthquakes as self-organized critical phenomena, J. Geophys. Res., 94, 15,635-15,637, 1989.

Bakun, W. H., Seismic activity of the southern Calaveras fault in central California, Bull. Seismol. Soc. Am., 70, 1181-1197, 1980.

Biegel, R. L., C. G. Sammis, and J. H. Dieterich, The frictional properties of a simulated gouge having a fractal particle distribution, J. Struct. Geol., 11, 827-846, 1989.

Blanpied, M. L., D. A. Lockner, and J. D. Byerlee, Fault stability inferred from granite sliding experiments at hydrothermal conditions, Geophys. Res. Lett., 18(4), 609-612, 1991.

Brown, S., J. B. Rundle, and C. H. Scholz, A simplified springblock model of earthquakes, Geophys. Res. Lett., 18, 215-218, 1991.

Burridge, R., and L. Knopoff, Model and theoretical seismicity, Bull. Seismol. Soc. Am., 57, 341-371, 1967.

Cao, T., and K. Aki, Seismicity simulation with a rate- and state-dependent friction law, Pure Appl. Geophys., 124, 487-514, 1986.

Carlson, J. M., A two dimensional model of a fault, Phys. Rev. A, 44, 6226-6232, 1991.

Carlson, J. M., and J. S. Langer, Mechanical model of an earthquake, Phys. Rev. A Gen. Phys., 40, 6470-6484, 1989.

Carlson, J. M., J. S. Langer, B. Shaw, and C. Tang, Intrinsic properties of a Burridge-Knopoff model of a fault, Phys. Rev. A, 44, 884-897, 1991.

Chen, K., P. Bak, and S. P. Obukhov, Self-organized criticality in a crack-propagation model of earthquakes, Phys. Rev. A, 43, 625630, 1991.

Chinnery, M., The stress changes that accompany strike-slip faulting, Bull. Seismol. Soc. Am., 53, 921-932, 1963.

Christensen, K., and Z. Olami, Variation of the Gutenberg-Richter $b$ values and nontrivial temporal correlations in a spring-block model for earthquakes, J. Geophys. Res., 97, 8729-8735, 1992.

de Sousa Vieira, M., G. L. Vasconcelos, and S. R. Nagel, Dynamics of spring-block models: Tuning to criticality, Phys. Rev. E, 47, R2221-R2224, 1993.

Dieterich, J. H., Constitutive properties of faults with simulated gouge, in Mechanical Behavior of Crustal Rocks: The Handin Volume, Geophys. Monogr. Ser., vol. 24, edited by N. L. Carter et al., pp. 108-120, AGU, Washington, D. C., 1981.

Golub, G. H., and C. F. VanLoan, Matrix Computations, Johns Hopkins University Press, Baltimore, Md., 1983.

Gu, J.-C., J. R. Rice, A. L. Ruina, and S. T. Tse, Slip motion and stability of a single degree of freedom elastic system with rate and state dependent friction, J. Mech. Phys. Solids, 32, 167-196, 1984.

Hirth, J. P., and J. Lothe, Theory of Dislocations, McGraw-Hill, New York, 1968.

Horowitz, F. G., and A. Ruina, Slip patterns in a spatially homogeneous fault model, J. Geophys. Res., 94, 10,279-10,298, 1989.

Ito, K., and M. Matsuzaki, Earthquakes as self-organized critical phenomena, J. Geophys. Res., 95, 6853-6860, 1990.

King, G. C. P., Speculations on the geometry of the initiation and termination processes of earthquake rupture and its relation to morphology and geological structure, Pure Appl. Geophys., 124, 567-585, 1986.

King, G., and J. Nabelek, Role of fault bends in the initiation and termination of earthquake rupture, Science, 228, 984-987, 1985.

Knopoff, L., J. A. Landoni, and M. S. Abinante, Dynamical model of an earthquake fault with localization, Phys. Rev. A, 46, 7445-7449, 1992.

Kostrov, B. V., and S. Das, Principles of Earthquake Source Mechanics, Cambridge University Press, New York, 1988.

Lindh, A. G., and D. M. Boore, Control of rupture by fault geometry during the 1966 Parkfield earthquake, Bull. Seismol. Soc. Am., 71, 95-116, 1981.

Madariaga, R., Dynamics of an expanding circular fault, Bull. Seismol. Soc. Am., 66, 639-666, 1976.

Madariaga, R., and A. Cochard, Heterogeneous faulting and friction, in Proceedings of International Symposium on Earthquake Disaster Prevention, May 1992, vol. I, edited by R. Melli, pp. 103-118, Mexico City, CENAPRED, 1992. 
Narkounskaia, G., J. Huang, and D. Turcotte, Chaotic and selforganized critical behavior of a generalized slider-block model, $J$. Stat. Phys., 67, 1151-1183, 1992.

Power, W. L., and T. E. Tullis, Euclidian and fractal models for the description of rock surface roughness, J. Geophys. Res., 96, 415-424, 1991.

Power, W. L., T. E. Tullis, and J. D. Weeks, Roughness and wear during brittle faulting, J. Geophys. Res., 93, 15,268-15,278, 1988.

Rice, J. R., The mechanics of earthquake rupture, in Physics of the Earth's Interior, edited by A. Dziewonski and E. Boschi, pp. 555-649, North-Holland, New York, 1980.

Rice, J. R., Spatio-temporally complex fault slip: 3D simulations with rate- and state-dependent friction on a fault surface between elastically deformable continua (abstract), Eos Trans. AGU, 72(17), Spring Meeting suppl., 278, 1991.

Rice, J. R., Fault stress states, pore pressure distributions, and the weakness of the San Andreas Fault, in Fault Mechanics and Transport Properties in Rocks, edited by B. Evans and T.-F. Wong, pp. 475-503, Academic, San Diego, Calif., 1992.

Rice, J. R., and A. L. Ruina, Stability of steady frictional slipping, J. Appl. Mech., 50, 343-349, 1983.

Rice, J. R., and S. T. Tse, Dynamic motion of a single degree of freedom system following a rate and state dependent friction law, J. Geophys. Res., 91, 521-530, 1986.

Ruina, A. L., Slip instability and state variable friction laws, $J$. Geophys. Res., 88, 10,359-10,370, 1983.

Rundle, J. B., A physical model for earthquakes, 1, Fluctuations and interactions, J. Geophys. Res., 93, 6237-6254, $1988 a$.

Rundle, J. B., A physical model for earthquakes, 2, Application to southern California, J. Geophys. Res., 93, 6255-6274, $1988 b$.

Rundle, J. B., and H. Kanamori, Application of an inhomogeneous stress (patch) model to complex subduction zone earthquakes: A discrete interaction matrix approach, J. Geophys. Res., 92, 2606$2616,1987$.
Sacks, S. I., and P. A. Rydelek, Quantum earthquake concept (abstract), Seismol. Res. Lett., 63(1), 76, 1992.

Scholz, C. H., The critical slip distance for seismic faulting, Nature, 326, 761-763, 1988.

Schwartz, D. P., and K. J. Coppersmith, Fault behavior and characteristic earthquakes: Examples from the Wasatch and San Andreas faults, J. Geophys. Res., 89, 5681-5698, 1984.

Sibson, R. H., Earthquakes and lineament infrastructure, Philos. Trans. R. Soc. London, Ser. A, 317, 63-79, 1986.

Stuart, W. D., Forecast model for large and great earthquakes in southern California, J. Geophys. Res., 91, 13,771-13,786, 1986.

Tse, S. T., and J. R. Rice, Crustal earthquake instability in relation to the depth variation of frictional slip properties, J. Geophys. Res., 91, 9452-9472, 1986.

Vasconcelos, G. I., M. de Sousa Vieira, and S. R. Nagel, Phase transitions in a spring-block model of earthquakes, Physica $A$ $191,69-74,1992$.

Ward, S. N., A synthetic seismicity model for the Middle American Trench, J. Geophys. Res., 96, 21,433-21,442, 1991.

Wesnousky, S., Seismological and structural evolution of strike-slip faults, Nature, 335, 6188-6190, 1988.

Wesnousky, S., Seismicity as a function of cumulative geologic offset: Some observations from southern California, Bull. Seismol. Soc. Am., 80, 1374-1381, 1990.

Wong, T. F., Shear fracture energy of Westerly granite from post-failure behavior, J. Geophys. Res., 87, 990-1000, 1982.

J. R. Rice, Department of Earth and Planetary Sciences, Harvard University, 224 Pierce Hall, 29 Oxford Street, Cambridge, MA 02138.

(Received June 19, 1992

revised December 4, 1992;

accepted December 14, 1992.) 